\title{
The effects of introducing sterically demanding aryl substituents in $\left[\mathrm{Cu}\left(\mathrm{N}^{\wedge} \mathbf{N}\right)\left(P^{\wedge} \mathbf{P}\right)\right]^{+}$complexes
}

\author{
Fabian Brunner, ${ }^{a}$ Stefan Graber, ${ }^{a}$ Yann Baumgartner, ${ }^{a}$ Daniel Häussinger, ${ }^{a}$ \\ Alessandro Prescimone, ${ }^{a}$ Edwin C. Constable ${ }^{a}$ and Catherine E. Housecroft* ${ }^{a}$
}

Received 00th January 2012,

Accepted 00th January 2012

DOI: $10.1039 / \times 0 \times x 00000 x$

www.rsc.org/

\begin{abstract}
The syntheses and characterizations of six $\left[\mathrm{Cu}\left(\mathrm{N}^{\wedge} \mathrm{N}\right)(\mathrm{POP})\right]\left[\mathrm{PF}_{6}\right]$ and $\left[\mathrm{Cu}\left(\mathrm{N}^{\wedge} \mathrm{N}\right)(\right.$ xantphos $\left.)\right]\left[\mathrm{PF}_{6}\right]$ compounds $(\mathrm{POP}=\operatorname{bis}(2-($ diphenylphosphino)phenyl)ether, $\quad$ xantphos $=4,5$-bis(diphenylphosphino)-9,9dimethylxanthene) in which $\mathrm{N}^{\wedge} \mathrm{N}$ is a bpy ligand (1-Naphbpy, 2-Naphbpy, 1-Pyrbpy) bearing a sterically hindered 1-naphthyl, 2-naphthyl or 1-pyrenyl substituent in the 6-position are reported. Single-crystal structure determinations of five complexes confirm a distorted tetrahedral environment for copper(I) and a preference for the $\mathrm{N}^{\wedge} \mathrm{N}$ ligand to be oriented with the sterically-demanding aryl group being remote from the $\left(\mathrm{C}_{6} \mathrm{H}_{4}\right)_{2} \mathrm{O}$ unit of POP or the xanthene 'bowl' of xantphos. The angle between the ring planes of the bpy range from 5.8 to $26.0^{\circ}$ and this is associated with interactions between the aryl unit and the phenyl substituents of the $\mathrm{P}^{\wedge} \mathrm{P}$ ligand. In solution at room temperature, the complexes undergo dynamic behaviour which has been investigated using variable temperature $2 \mathrm{D}$ NMR spectroscopy. The $\left[\mathrm{Cu}\left(\mathrm{N}^{\wedge} \mathrm{N}\right)(x a n t p h o s)\right]^{+}$complexes exist as a mixture of conformers which interconvert through inversion of the xanthene bowl-shaped unit; the preferencce for one conformer over the other is significantly changed on going from $N^{\wedge} N=$ Phbpy to 1-Pyrbpy (Phbpy = 6-phenyl-2,2'-bipyridine). The electrochemical and photophysical properties of the $\left[\mathrm{Cu}\left(\mathrm{N}^{\wedge} \mathrm{N}\right)(\mathrm{POP})\right]\left[\mathrm{PF}_{6}\right]$ and $\left[\mathrm{Cu}\left(\mathrm{N}^{\wedge} \mathrm{N}\right)(\mathrm{xantphos})\right]\left[\mathrm{PF}_{6}\right]$ compounds are presented; the compounds are orange emitters but the introduction of the 1-naphthyl, 2-naphthyl or 1pyrenyl substituents result in poor photoluminescence quantum yields.
\end{abstract}

\section{Introduction}

In the last few years, there has been increased interest in harnessing the emissive properties of copper(I) complexes in low-cost, light-emitting electrochemical cells (LECs). ${ }^{1,2,3}$ Building upon the pioneering work of McMillan and coworkers, ${ }^{4,5}\left[\mathrm{Cu}\left(\mathrm{N}^{\wedge} \mathrm{N}\right)(\mathrm{POP})\right]^{+}$and $\left[\mathrm{Cu}\left(\mathrm{N}^{\wedge} \mathrm{N}\right)(\text { xantphos })\right]^{+}$ complexes $(\mathrm{POP}=\operatorname{bis}(2-($ diphenylphosphino)phenyl)ether, xantphos = 4,5-bis(diphenylphosphino)-9,9-dimethylxanthene, $\mathrm{N}^{\wedge} \mathrm{N}$ is an $N, N^{\prime}$-chelating ligand) are established as favoured components in the emissive layers in $\operatorname{LECs}^{6,7,8,9,10,11,12,13,14}$ or organic light-emitting devices (OLEDs). ${ }^{15,16}$ Related complexes find application in oxygen sensing, ${ }^{17} \mathrm{CO}_{2}$ reduction, ${ }^{18}$ and water reduction. ${ }^{19,20}$ The $\mathrm{N}^{\wedge} \mathrm{N}$ ligand in $\left[\mathrm{Cu}\left(\mathrm{N}^{\wedge} \mathrm{N}\right)(\mathrm{POP})\right]^{+}$and $\left[\mathrm{Cu}\left(\mathrm{N}^{\wedge} \mathrm{N}\right)(\text { xantphos) }]^{+}\right.$is usually a 2,2'-bipyridine (bpy) or 1,10-phenanthroline (phen) derivative, and the introduction of simple substituents into the 6- and 6'-positions of bpy or 2- and 9 -positions of phen modulates the emission properties of the complexes. $^{4,7,8}$

We recently demonstrated the remarkable performance of $\left[\mathrm{Cu}\left(\mathrm{N}^{\wedge} \mathrm{N}\right)(\mathrm{POP})\right]\left[\mathrm{PF}_{6}\right]$ and $\left[\mathrm{Cu}\left(\mathrm{N}^{\wedge} \mathrm{N}\right)(\right.$ xantphos $\left.)\right]\left[\mathrm{PF}_{6}\right]\left(\mathrm{N}^{\wedge} \mathrm{N}=\right.$ 6-methyl-2,2'-bipyridine, 6-ethyl-2,2'-bipyridine or 6,6'- dimethyl-2,2'-bipyridine) in LECs. ${ }^{8}$ However, achieving both high efficacy and long device lifetime remains challenging. A LEC with $[\mathrm{Cu}(\mathrm{Mebpy})(\mathrm{xantphos}))]\left[\mathrm{PF}_{6}\right]$ in the emissive layer had a lifetime of $>15 \mathrm{~h}$ and an efficacy of $1.9 \mathrm{~cd} \mathrm{~A}^{-1}$. The latter increased to $3.0 \quad \mathrm{~cd} \quad \mathrm{~A}^{-1}$ on going to $\left[\mathrm{Cu}\left(\mathrm{Me}_{2}\right.\right.$ bpy)(xantphos) $]\left[\mathrm{PF}_{6}\right]$, but the lifetime decreased to $1 \mathrm{~h}$. Introducing an ethyl group in place of the 6-methyl substituent led to a significant increase in lifetime $(>40 \mathrm{~h})$ but at the expense of luminance. ${ }^{8}$ Since POP and xantphos are both sterically demanding, there are significant constraints on the substituents that can be introduced into the 6- and 6'-positions of the bpy ligand. Ligand redistribution reactions which lead to the equilibrium mixtures of $\left[\mathrm{Cu}\left(\mathrm{N}^{\wedge} \mathrm{N}\right)\left(\mathrm{P}^{\wedge} \mathrm{P}\right)\right]^{+},\left[\mathrm{Cu}\left(\mathrm{N}^{\wedge} \mathrm{N}\right)_{2}\right]^{+}$and $\left[\mathrm{Cu}\left(\mathrm{P}^{\wedge} \mathrm{P}\right)_{2}\right]^{+}$are a recognized problem. ${ }^{21}$ Nevertheless, the tetrahedral copper(I) coordination sphere in $\left[\mathrm{Cu}\left(\mathrm{N}^{\wedge} \mathrm{N}\right)(\mathrm{POP})\right]^{+}$ and $\left[\mathrm{Cu}\left(\mathrm{N}^{\wedge} \mathrm{N}\right)(\text { xantphos) }]^{+}\right.$is able to accommodate a 6-phenyl2,2'-bipyridine (Phbpy) ligand giving complexes which are stable in $\mathrm{CH}_{2} \mathrm{Cl}_{2}$ solutions. ${ }^{8}$ While both $[\mathrm{Cu}(\mathrm{Phbpy})(\mathrm{POP})]^{+}$and $[\mathrm{Cu}(\mathrm{Phbpy})(\text { xantphos })]^{+}$exhibit poor photoluminescence quantum yields, ${ }^{8}$ the compounds remain of interest from a structural viewpoint. In the solid-state, the orientation of the Phbpy ligand in $[\mathrm{Cu}(\mathrm{Phbpy})(\mathrm{POP})]^{+}$and $[\mathrm{Cu} \text { (Phbpy)(xantphos) }]^{+}$(Fig. 1a) is rotated $\sim 180^{\circ}$ with respect 
to that in $[\mathrm{Cu}(\mathrm{Mebpy})(\mathrm{xantphos})]^{+} \quad$ (Fig. 1b), $\left[\mathrm{Cu}(\text { Etbpy)(POP) }]^{+}\right.$and $[\mathrm{Cu}(\text { Etbpy)(xantphos })]^{+}$, and in solution two conformers of $[\mathrm{Cu} \text { (xantphos)(Phbpy) }]^{+}$exist with approximately equal populations. ${ }^{8}$ These conformers are related by the inversion of the xanthene unit which has a 'bowl'-like structure (Fig. 1). We therefore decided to extend the investigation to larger aryl substituents to investigate the effect on solution dynamics and solid-state structure. The $\mathrm{N}^{\wedge} \mathrm{N}$ ligands selected for the current study are shown in Scheme 1.

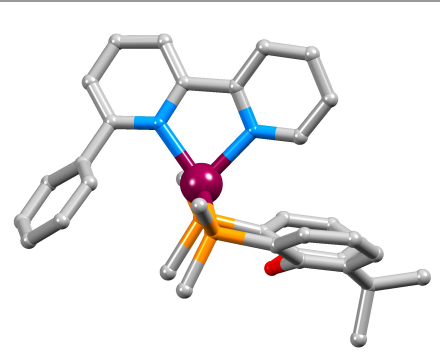

(a)

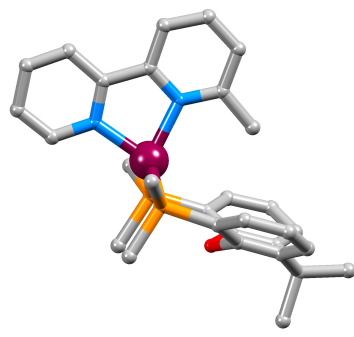

(b)

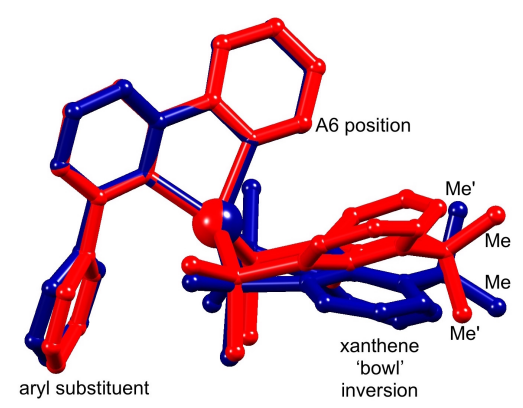

(c)

Fig. 1. Structures of (a) $[\mathrm{Cu}(\mathrm{Mebpy})(\mathrm{xantphos})]^{+}$and (b) $[\mathrm{Cu}(\mathrm{Phbpy}) \text { (xantphos) }]^{+}$ (c) Overlay of the structures of two conformers of [Cu(xantphos)(Phbpy)]+ which are related by inversion of the xanthene unit). Only the ipso-C atoms of each $\mathrm{PPh}_{2}$ phenyl ring is shown and $\mathrm{H}$ atoms are omitted. The relevance of the 'A6 position' and the positions of the methyl groups (Me and Me') are discussed later in the paper. Data for the figure are from ref. ${ }^{8}$.

\section{Experimental}

General. Microwave reactions were performed in a Biotage Initiator 8 reactor. ${ }^{1} \mathrm{H},{ }^{13} \mathrm{C}$ and ${ }^{31} \mathrm{P}$ NMR spectra were recorded using Bruker Avance III-400, 500 and 600 NMR spectrometers; spectra were recorded at $\sim 295 \mathrm{~K}$ unless otherwise stated. ${ }^{1} \mathrm{H}$ and ${ }^{13} \mathrm{C}$ NMR chemical shifts were referenced to the residual solvent peaks with respect to $\delta(\mathrm{TMS})=0 \mathrm{ppm}$ and ${ }^{31} \mathrm{P} \mathrm{NMR}$ chemical shifts with respect to $\delta\left(85 \%\right.$ aqueous $\left.\mathrm{H}_{3} \mathrm{PO}_{4}\right)=0 \mathrm{ppm}$. Solution absorption spectra were measured using an Agilent 8453 spectrophotometer. A Bruker esquire 3000plus instrument was used to record electrospray ionization (ESI) mass spectra. Quantum yields were measured using a Hamamatsu absolute photoluminescence (PL) quantum yield spectrometer C11347 Quantaurus-QY. Emission lifetimes and powder emission spectra were measured with a Hamamatsu Compact Fluorescence lifetime Spectrometer C11367 Quantaurus-Tau, using an LED light source with $\lambda_{\mathrm{exc}}=365 \mathrm{~nm}$.

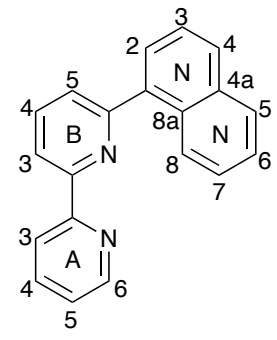

1-Naphpby

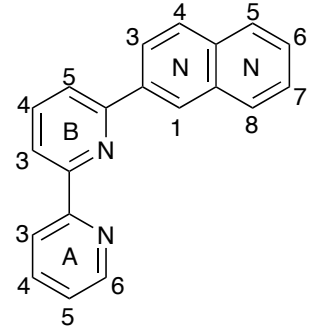

2-Naphpby

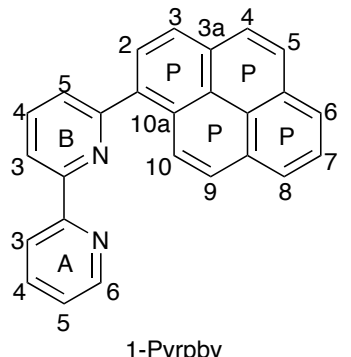

1-Pyrpby

Scheme 1. Structures of the $\mathrm{N}^{\wedge} \mathrm{N}$ ligands with ring and atom labelling for $\mathrm{NMR}$ spectroscopic assignments.

Electrochemical measurements were carried out using a $\mathrm{CH}$ Instruments $900 \mathrm{~B}$ potentiostat with $\left[\mathrm{Bu}_{4} \mathrm{~N}\right]\left[\mathrm{PF}_{6}\right](0.1 \mathrm{M})$ as supporting electrolyte and at a scan rate of $0.1 \mathrm{~V} \mathrm{~s}^{-1}$. The working electrode was glassy carbon, the reference electrode was a leakless $\mathrm{Ag}^{+} / \mathrm{AgCl}$ (eDAQ ET069-1) and the counterelectrode platinum wire. Final potentials were referenced with respect to the $\mathrm{Fc} / \mathrm{Fc}^{+}$couple.

6-Bromo-2,2'-bipyridine was prepared according to a literature procedure ${ }^{22}$ or by the more convenient method described below. POP and xantphos were purchased from Fluorochem and SPhosPd G2 from Sigma-Aldrich. $\left[\mathrm{Cu}(\mathrm{MeCN})_{4}\right]\left[\mathrm{PF}_{6}\right]$ was prepared by the published method. ${ }^{23}$

6-Bromo-2,2'-bipyridine. 2,6-Dibromopyridine (1.27 g, 5.38 $\mathrm{mmol})$ and $\left[\mathrm{Pd}\left(\mathrm{PPh}_{3}\right)_{4}\right](311 \mathrm{mg}, 0.27 \mathrm{mmol})$ were placed in a 10-20 $\mathrm{ml}$ microwave vial. The vial was evacuated and refilled with $\mathrm{N}_{2}$ three times before dry THF $(5 \mathrm{ml})$ was added. $\mathrm{N}_{2}$ was bubbled through the solution for $20 \mathrm{~min}$ before a solution of 2pyridylzinc bromide $(0.5 \mathrm{M}$ in THF, $14.0 \mathrm{ml}, 7.00 \mathrm{mmol})$ was added and the vial was sealed. The reaction was performed in a microwave reactor $\left(90{ }^{\circ} \mathrm{C}, 2.5 \mathrm{~h}\right)$. Saturated aqueous $\mathrm{NaHCO}_{3}$ $(30 \mathrm{ml})$ was added and the crude mixture was extracted with $\mathrm{CH}_{2} \mathrm{Cl}_{2}(3 \times 70 \mathrm{ml})$. The combined organic layers were washed with water $(3 \times 180 \mathrm{ml})$ and dried over $\mathrm{MgSO}_{4}$. The solvent was removed in vacuo and the crude material was purified by column chromatography (Alox 90, hexane : ethyl acetate $50: 1$ ) to give 6-bromo-2,2'-bipyridine (462 $\mathrm{mg}, 2.39 \mathrm{mmol}, 44 \%$ ) as a white powder. ${ }^{1} \mathrm{H}$ NMR spectroscopic data were consistent with the literature data ${ }^{1}$. ESI MS: $m / z 234.7[\mathrm{M}+\mathrm{H}]^{+}$(base peak, calc. 235.0).

6-(Naphthalen-1-yl)-2,2'-bipyridine (1-Naphbpy). 6(Naphthalen-1-yl)-2,2'-bipyridine was prepared based on methodoloy described in the literature. ${ }^{24} \mathrm{~A}$ mixture of 6-bromo2,2'-bipyridine (325 mg, $1.38 \mathrm{mmol}$ ), 1-naphthaleneboronic 
acid (261 mg, $1.51 \mathrm{mmol}$ and SPhosPd G2 (49.7 mg, 0.07 $\mathrm{mmol})$ in toluene $(15 \mathrm{ml}), \mathrm{EtOH}(7 \mathrm{ml})$ and aqueous $\mathrm{Cs}_{2} \mathrm{CO}_{3}(2$ $\mathrm{M}, 1.5 \mathrm{ml}$ ) were heated at $80^{\circ} \mathrm{C}$ under inert conditions for $23 \mathrm{~h}$. The mixture was allowed to cool to room temperature before toluene $(30 \mathrm{ml})$ was added. The organic layer was washed with water $(3 \times 50 \mathrm{ml})$ dried over $\mathrm{MgSO}_{4}$ and filtered. The solvent was removed in vacuo and the product was purified by column chromatography (Alox 90, cyclohexane : ethyl acetate $25: 1$ ) to give 6-(naphthalen-1-yl)-2,2'-bipyridine (259 mg, $0.92 \mathrm{mmol}$, $67 \%$ ) as a white powder. ${ }^{1} \mathrm{H}$ NMR (500 MHz, DMSO- $\left.d_{6}\right)$ $\delta / \mathrm{ppm}$ : 8.74 (ddd, $\left.J=4.8,1.8,0.9 \mathrm{~Hz}, 1 \mathrm{H}, \mathrm{H}^{\mathrm{A} 6}\right), 8.47(\mathrm{dd}, J=$ $\left.7.9,1.0 \mathrm{~Hz}, 1 \mathrm{H}, \mathrm{H}^{\mathrm{B} 3}\right), 8.37\left(\mathrm{dt}, J=8.0,1.1 \mathrm{~Hz}, 1 \mathrm{H}, \mathrm{H}^{\mathrm{A} 3}\right), 8.17$ $\left(\mathrm{m}, 1 \mathrm{H}, \mathrm{H}^{\mathrm{N} 8}\right), 8.13\left(\mathrm{t}, J=7.8 \mathrm{~Hz}, 1 \mathrm{H}, \mathrm{H}^{\mathrm{B} 4}\right), 8.07-8.03$ (overlapping $\mathrm{m}, 2 \mathrm{H}, \mathrm{H}^{\mathrm{N} 4+\mathrm{N} 5}$ ), 7.93 (ddd, $J=8.0,7.5,1.8 \mathrm{~Hz}$, $\left.1 \mathrm{H}, \mathrm{H}^{\mathrm{A} 4}\right), 7.74\left(\mathrm{dd}, J=7.7,1.0 \mathrm{~Hz}, 1 \mathrm{H}, \mathrm{H}^{\mathrm{B} 5}\right), 7.72(\mathrm{dd}, J=7.1$, $\left.1.3 \mathrm{~Hz}, 1 \mathrm{H}, \mathrm{H}^{\mathrm{N} 2}\right), 7.64\left(\mathrm{dd}, J=8.2,7.1 \mathrm{~Hz}, 1 \mathrm{H}, \mathrm{H}^{\mathrm{N} 3}\right), 7.58$ (ddd, $\left.J=8.2,6.8,1.5 \mathrm{~Hz}, 1 \mathrm{H}, \mathrm{H}^{\mathrm{N} 6}\right), 7.54(\mathrm{ddd}, J=8.2,6.8,1.6$ $\mathrm{Hz}, 1 \mathrm{H}, \mathrm{H}^{\mathrm{N} 7}$ ), 8.74 (ddd, $\left.J=7.5,4.8,1.2 \mathrm{~Hz}, 1 \mathrm{H}, \mathrm{H}^{\mathrm{A} 5}\right) .{ }^{13} \mathrm{C}$ NMR (126 MHz, DMSO-d $\left.d_{6}\right) \delta /$ ppm: $157.9\left(\mathrm{C}^{\mathrm{B} 6}\right), 155.3\left(\mathrm{C}^{\mathrm{A} 2}\right)$, $154.9\left(\mathrm{C}^{\mathrm{B} 2}\right), 149.4\left(\mathrm{C}^{\mathrm{A} 6}\right), 138.2\left(\mathrm{C}^{\mathrm{B} 4}\right), 137.9\left(\mathrm{C}^{\mathrm{N} 1}\right), 137.3\left(\mathrm{C}^{\mathrm{A} 4}\right)$, $133.5\left(\mathrm{C}^{\mathrm{N} 4 \mathrm{a}}\right), 130.6\left(\mathrm{C}^{\mathrm{N} 8 \mathrm{a}}\right), 128.9\left(\mathrm{C}^{\mathrm{N} 4}\right), 128.4\left(\mathrm{C}^{\mathrm{N} 5}\right), 127.6$ $\left(\mathrm{C}^{\mathrm{N} 2}\right), 126.7\left(\mathrm{C}^{\mathrm{N} 7}\right), 126.0\left(\mathrm{C}^{\mathrm{N} 6}\right), 125.5\left(\mathrm{C}^{\mathrm{N} 3}\right), 125.3\left(\mathrm{C}^{\mathrm{N} 8}\right), 125.2$ $\left(\mathrm{C}^{\mathrm{B} 5}\right), 124.3\left(\mathrm{C}^{\mathrm{A} 5}\right), 120.6\left(\mathrm{C}^{\mathrm{A} 3}\right), 118.9\left(\mathrm{C}^{\mathrm{B} 3}\right)$. MALDI-TOF MS: $m / z 283.0[\mathrm{M}+\mathrm{H}]^{+}$(base peak, calc. 283.1). Found: $\mathrm{C} 84.78, \mathrm{H}$ 5.37, N 9.73; $\mathrm{C}_{20} \mathrm{H}_{14} \mathrm{~N}_{2}$ requires $\mathrm{C} 85.08, \mathrm{H} 5.00, \mathrm{~N} 9.92 \%$.

\section{6-(Naphthalen-2-yl)-2,2'-bipyridine}

(2-Naphbpy).

(Naphthalen-2-yl)-2,2'-bipyridine was prepared using the same procedure as for 6-(naphthalen-1-yl)-2,2'-bipyridine but starting with 6-bromo-2,2'-bipyridine (350 $\mathrm{mg}, 1.49 \mathrm{mmol}), 2-$ naphthaleneboronic acid (282 mg, $1.64 \mathrm{mmol})$ and SPhosPd G2 (53.6 $\mathrm{mg}, 0.07 \mathrm{mmol})$ in toluene $(15 \mathrm{ml})$, EtOH $(7 \mathrm{ml})$ and aqueous $\mathrm{Cs}_{2} \mathrm{CO}_{3}(2 \mathrm{M}, 1.7 \mathrm{ml})$. 6-(Naphthalen-2-yl)-2,2'bipyridine (288 $\mathrm{mg}, 1.02 \mathrm{mmol}, 69 \%$ ) was isolated as a white powder. ${ }^{1} \mathrm{H}$ and ${ }^{13} \mathrm{C}$ NMR data were consistent with the literature data. ${ }^{25}$ ESI MS: $m / z 305.0[\mathrm{M}+\mathrm{Na}]^{+}$(base peak, calc. 305.1). Found: $\mathrm{C} 84.73, \mathrm{H} \mathrm{5.48,} \mathrm{N} 10.11 ; \mathrm{C}_{20} \mathrm{H}_{14} \mathrm{~N}_{2}$ requires $\mathrm{C}$ 85.08, H 5.00, N 9.92\%.

6-(Pyrene-1-yl)-2,2'-bipyridine (1-Pyrbpy). 6-Bromo-2,2'bipyridine (1.50 g, $4.89 \mathrm{mmol})$, pyrene-1-boronic acid (1.32 g, $5.38 \mathrm{mmol})$ and $\mathrm{Na}_{2} \mathrm{CO}_{3}(1.04 \mathrm{~g}, 9.78 \mathrm{mmol})$ were dissolved in toluene $(200 \mathrm{ml})$ and water $(50 \mathrm{ml})$. The mixture was degassed with $\mathrm{N}_{2}$ for $50 \mathrm{~min}$ before $\left[\mathrm{Pd}\left(\mathrm{PPh}_{3}\right)_{4}\right](282 \mathrm{mg}, 0.24 \mathrm{mmol})$ was added. The reaction mixture was heated at reflux for $69 \mathrm{~h}$ under rigorous exclusion of light. The reaction mixture was cooled to room temperature and the aqueous phase was separated. The organic layer was washed with water $(50 \mathrm{ml})$, dried over $\mathrm{MgSO}_{4}$ and evaporated to dryness. The crude product was purified by column chromatography (Alox 90, hexane : $\mathrm{CH}_{2} \mathrm{Cl}_{2} 4: 1$ to pure $\mathrm{CH}_{2} \mathrm{Cl}_{2}$, then Silica 60 , toluene : ethyl acetate $10: 1$ to 5:1, then Alox 90, pure $\mathrm{CH}_{2} \mathrm{Cl}_{2}$, the Silica 60 pure $\mathrm{CH}_{2} \mathrm{Cl}_{2}$ to $\mathrm{CH}_{2} \mathrm{Cl}_{2}$ : ethyl acetate $\left.10: 1\right)$ to give 6(pyrene-1-yl)-2,2'-bipyridine (1.25 g, $3.51 \mathrm{mmol}, 72 \%)$ as a yellow powder. ${ }^{1} \mathrm{H}$ NMR (500 MHz, acetone- $\left.\mathrm{d}_{6}\right) \delta / \mathrm{ppm}: 8.75$ (ddd, $J=4.8,1.8,0.9 \mathrm{~Hz}, 1 \mathrm{H}, \mathrm{H}^{\mathrm{A} 6}$ ), 8.62 (overlapping dd, $J=$ $7.9,0.9 \mathrm{~Hz}, 1 \mathrm{H}, \mathrm{H}^{\mathrm{B} 6}$ ), 8.62 (overlapping $\mathrm{d}, J=9.1 \mathrm{~Hz}, 1 \mathrm{H}$, $\left.\mathrm{H}^{\mathrm{P} 10}\right), 8.59\left(\mathrm{~d}, J=8.0 \mathrm{~Hz}, 1 \mathrm{H}, \mathrm{H}^{\mathrm{A} 3}\right), 8.42(\mathrm{~d}, J=7.9 \mathrm{~Hz}, 1 \mathrm{H}$,
$\left.\mathrm{H}^{\mathrm{P} 3}\right), 8.34\left(\mathrm{~d}, J=7.6 \mathrm{~Hz}, 1 \mathrm{H}, \mathrm{H}^{\mathrm{P} 6}\right), 8.33(\mathrm{~d}, J=7.9 \mathrm{~Hz}, 1 \mathrm{H}$, $\left.\mathrm{H}^{\mathrm{P} 2}\right), 8.31\left(\mathrm{~d}, J=7.6 \mathrm{~Hz}, 1 \mathrm{H}, \mathrm{H}^{\mathrm{P} 8}\right), 8.26\left(\mathrm{~s}, 2 \mathrm{H}, \mathrm{H}^{\mathrm{P} 4+\mathrm{P} 5}\right), 8.22(\mathrm{~d}$, $\left.J=9.1 \mathrm{~Hz}, 1 \mathrm{H}, \mathrm{H}^{\mathrm{P} 9}\right), 8.18\left(\mathrm{t}, J=7.8 \mathrm{~Hz}, 1 \mathrm{H}, \mathrm{H}^{\mathrm{B} 4}\right), 8.11(\mathrm{t}, J=$ $\left.7.6 \mathrm{~Hz}, 1 \mathrm{H}, \mathrm{H}^{\mathrm{P} 7}\right), 7.93\left(\mathrm{td}, J=7.8,1.8 \mathrm{~Hz}, 1 \mathrm{H}, \mathrm{H}^{\mathrm{A} 4}\right), 7.91(\mathrm{dd}, J$ $\left.=7.6,0.9 \mathrm{~Hz}, 1 \mathrm{H}, \mathrm{H}^{\mathrm{B} 5}\right), 7.45(\mathrm{ddd}, J=7.5,4.8,1.2 \mathrm{~Hz}, 1 \mathrm{H}$, $\left.\mathrm{H}^{\mathrm{A} 5}\right) .{ }^{13} \mathrm{C}$ NMR $\left(126 \mathrm{MHz}\right.$, acetone-d $\left.\mathrm{d}_{6}\right) \delta / \mathrm{ppm}: 159.8\left(\mathrm{C}^{\mathrm{B} 6}\right)$, $156.9\left(\mathrm{C}^{\mathrm{A} 2}\right), 156.7\left(\mathrm{C}^{\mathrm{B} 2}\right), 150.2\left(\mathrm{C}^{\mathrm{A} 6}\right), 138.7\left(\mathrm{C}^{\mathrm{B} 4}\right), 137.9\left(\mathrm{C}^{\mathrm{A} 4}\right)$, $132.4\left(\mathrm{C}^{\mathrm{P} 5}\right), 131.9\left(\mathrm{C}^{\mathrm{P} 8 \mathrm{a}}\right), 129.6\left(\mathrm{C}^{\mathrm{P} 10 \mathrm{c}}\right), 128.8\left(\mathrm{C}^{\mathrm{P9}}\right), 128.7$ $\left(\mathrm{C}^{\mathrm{P} 2}\right), 128.6\left(\mathrm{C}^{\mathrm{P} 4+\mathrm{P} 5}\right), 128.4\left(\mathrm{C}^{\mathrm{P} 1}\right), 127.2\left(\mathrm{C}^{\mathrm{P} 7}\right), 126.6\left(\mathrm{C}^{\mathrm{B} 5}\right)$, $126.4\left(\mathrm{C}^{\mathrm{P} 6}\right), 126.1\left(\mathrm{C}^{\mathrm{A} 10 \mathrm{a}}\right), 126.1\left(\mathrm{C}^{\mathrm{P} 8}\right), 126.0\left(\mathrm{C}^{\mathrm{P} 10}\right), 125.8$ $\left(\mathrm{C}^{\mathrm{P} 10 \mathrm{~b}}\right), 125.7\left(\mathrm{C}^{\mathrm{P} 3}\right), 125.6\left(\mathrm{C}^{\mathrm{P} 3 \mathrm{a}}\right), 125.0\left(\mathrm{C}^{\mathrm{A} 5}\right), 121.7\left(\mathrm{C}^{\mathrm{A} 3}\right)$, $119.8\left(\mathrm{C}^{\mathrm{B} 3}\right)$. ESI MS: m/z $357.1[\mathrm{M}+\mathrm{H}]^{+}$(base peak, calc. 357.1). Found: $\mathrm{C} 87.37, \mathrm{H} 4.48, \mathrm{~N} 7.50 ; \mathrm{C}_{26} \mathrm{H}_{16} \mathrm{~N}_{2}$ requires $\mathrm{C}$ 87.62, H 4.52, N 7.86\%.

[Cu(1-Naphbpy)(POP)][PF 6 . $\left[\mathrm{Cu}(\mathrm{MeCN})_{4}\right]\left[\mathrm{PF}_{6}\right](93.2 \mathrm{mg}$, $0.25 \mathrm{mmol})$ and POP (135 $\mathrm{mg}, 0.25 \mathrm{mmol})$ were dissolved in $\mathrm{CH}_{2} \mathrm{Cl}_{2}(30 \mathrm{ml})$ and the mixture was stirred for $2 \mathrm{~h}$ at room temperature. 1-Naphbpy (70.6 $\mathrm{mg}, 0.25 \mathrm{mmol})$ was added and stirring was continued for $2 \mathrm{~h}$. The yellow solution was filtered and the solvent was removed from the filtrate under vacuum. The solid product was washed with hexane $(2 \times 30 \mathrm{ml})$ and dried under vacuum. [Cu(1-Naphbpy)(POP)][PF 6 (244 mg, $0.21 \mathrm{mmol}, 82 \%)$ was isolated as a yellow solid. ${ }^{1} \mathrm{H}$ NMR (500 $\mathrm{MHz}$, acetone- $\left.d_{6}, 253 \mathrm{~K}\right) \delta / \mathrm{ppm}: 8.80(\mathrm{dd}, J=8.1,1.1 \mathrm{~Hz}, 1 \mathrm{H}$, $\left.\mathrm{H}^{\mathrm{B} 3}\right), 8.72\left(\mathrm{dt}, J=8.3,1.0 \mathrm{~Hz}, 1 \mathrm{H}, \mathrm{H}^{\mathrm{A} 3}\right), 8.34(\mathrm{t}, J=7.9 \mathrm{~Hz}$, $\left.1 \mathrm{H}, \mathrm{H}^{\mathrm{B} 4}\right), 8.17\left(\mathrm{dd}, J=5.2,0.9 \mathrm{~Hz}, 1 \mathrm{H}, \mathrm{H}^{\mathrm{A} 6}\right), 8.13-8.06$ (overlapping $\left.\mathrm{m}, 3 \mathrm{H}, \mathrm{H}^{\mathrm{A} 4+\mathrm{N} 4+\mathrm{N} 6}\right), 7.62(\mathrm{dd}, J=7.7,1.0 \mathrm{~Hz}, 1 \mathrm{H}$, $\mathrm{H}^{\mathrm{B} 5}$ ), 7.54-7.49 (overlapping $\mathrm{m}, 2 \mathrm{H}, \mathrm{H}^{\mathrm{C5} \mathrm{a}+\mathrm{N} 7}$ ), $7.45(\mathrm{td}, J=7.4$, $1.3 \mathrm{~Hz}, 1 \mathrm{H}, \mathrm{H}^{\mathrm{D} 4 \mathrm{a}}$ ), 7.42-7.39 (overlapping $\mathrm{m}, 2 \mathrm{H}, \mathrm{H}^{\mathrm{D} 4 \mathrm{c}+\mathrm{D} 4 \mathrm{~d}}$ ), $7.36\left(\mathrm{~m}, \quad 1 \mathrm{H}, \quad \mathrm{H}^{\mathrm{C} 6 \mathrm{a}}\right.$ ), 7.30-7.22 (overlapping $\mathrm{m}, \quad 5 \mathrm{H}$, $\mathrm{H}^{\mathrm{D} 3 \mathrm{a}+\mathrm{C} 5 \mathrm{~b}+\mathrm{D} 3 \mathrm{c}}$ ), 7.20-7.14 (overlapping $\mathrm{m}, 4 \mathrm{H}, \mathrm{H}^{\mathrm{N2}+\mathrm{D} 3 \mathrm{~d}+\mathrm{D} 4 \mathrm{~b}}$ ), 7.14$7.08 \quad$ (overlapping $\left.\mathrm{m}, \quad 4 \mathrm{H}, \quad \mathrm{H}^{\mathrm{A} 5+\mathrm{C} 4 \mathrm{a}+\mathrm{C} 6 \mathrm{~b}+\mathrm{N} 5}\right), \quad 7.01-6.93$ (overlapping $\mathrm{m}, 4 \mathrm{H}, \mathrm{H}^{\mathrm{C} 4 \mathrm{~b}+\mathrm{D} 2 \mathrm{c}+\mathrm{N} 8}$ ), 6.79-6.69 (overlapping $\mathrm{m}$, $\left.6 \mathrm{H}, \mathrm{H}^{\mathrm{C} 3 \mathrm{a}+\mathrm{D} 2 \mathrm{a}+\mathrm{D} 3 \mathrm{~b}+\mathrm{N} 3}\right), 6.53\left(\mathrm{~m}, 2 \mathrm{H}, \mathrm{H}^{\mathrm{D} 2 \mathrm{~d}}\right), 6.20(\mathrm{td}, J=7.8,1.6$ $\left.\mathrm{Hz}, 1 \mathrm{H}, \mathrm{H}^{\mathrm{C} 3 \mathrm{~b}}\right), 6.08\left(\mathrm{~m}, 2 \mathrm{H}, \mathrm{H}^{\mathrm{D} 2 \mathrm{~b}}\right) .{ }^{13} \mathrm{C} \mathrm{NMR}(126 \mathrm{MHz}$, acetone- $\left.d_{6}, 253 \mathrm{~K}\right) \delta / \mathrm{ppm}$ : $159.5\left(\mathrm{C}^{\mathrm{B} 6}\right), 157.8\left(\mathrm{C}^{\mathrm{Cla}}\right), 157.4$ $\left(\mathrm{C}^{\mathrm{Clb}}\right), 154.1\left(\mathrm{C}^{\mathrm{B} 2}\right), 153.0\left(\mathrm{C}^{\mathrm{A} 2}\right), 149.3\left(\mathrm{C}^{\mathrm{A} 6}\right), 139.2\left(\mathrm{C}^{\mathrm{A} 4}\right)$, $139.0\left(\mathrm{C}^{\mathrm{B} 4}\right), 138.8\left(\mathrm{C}^{\mathrm{N} 1}\right), 135.5\left(\mathrm{C}^{\mathrm{C} 3 \mathrm{a}}\right), 134.4\left(\mathrm{C}^{\mathrm{C} 3 \mathrm{~b}}\right), 134.4$ $\left(\mathrm{C}^{\mathrm{D} 2 \mathrm{a}}\right), 134.3\left(\mathrm{C}^{\mathrm{N} 4 \mathrm{a}}\right), 133.6\left(\mathrm{C}^{\mathrm{D} 2 \mathrm{c}}\right), 133.5\left(\mathrm{C}^{\mathrm{D} 2 \mathrm{~b}}\right), 132.9\left(\mathrm{C}^{\mathrm{D} 2 \mathrm{~d}}\right)$, $132.8\left(\mathrm{C}^{\mathrm{C} 5 \mathrm{a}}\right), 132.5\left(\mathrm{C}^{\mathrm{C} 5 \mathrm{~b}}\right), 131.0\left(\mathrm{C}^{\mathrm{N} 8 \mathrm{a}}\right), 130.6\left(\mathrm{C}^{\mathrm{D} 4 \mathrm{a}}\right), 130.5$ $\left(\mathrm{C}^{\mathrm{D} 4 \mathrm{c}+\mathrm{D} 4 \mathrm{~d}}\right), 130.4\left(\mathrm{C}^{\mathrm{D} 4 \mathrm{~b}}\right), 130.0\left(\mathrm{C}^{\mathrm{N} 4}\right), 129.4\left(\mathrm{C}^{\mathrm{B} 5}\right), 129.4\left(\mathrm{C}^{\mathrm{D} 3 \mathrm{c}}\right)$, $129.3\left(\mathrm{C}^{\mathrm{D} 3 \mathrm{a}}\right), 129.2\left(\mathrm{C}^{\mathrm{D} 3 \mathrm{~d}}\right), 129.0\left(\mathrm{C}^{\mathrm{N} 6}\right), 128.9\left(\mathrm{C}^{\mathrm{D} 3 \mathrm{~b}}\right), 127.8$ $\left(\mathrm{C}^{\mathrm{N} 5}\right), 127.8\left(\mathrm{C}^{\mathrm{N} 8}\right), 127.7\left(\mathrm{C}^{\mathrm{N} 2}\right), 127.0\left(\mathrm{C}^{\mathrm{N} 7}\right), 126.4\left(\mathrm{C}^{\mathrm{N} 3}\right), 126.0$ $\left(\mathrm{C}^{\mathrm{A} 5}\right), 125.8\left(\mathrm{C}^{\mathrm{C} 2 \mathrm{a}}\right), 125.6\left(\mathrm{C}^{\mathrm{C} 4 \mathrm{~b}}\right), 125.5\left(\mathrm{C}^{\mathrm{C} 4 \mathrm{a}}\right), 124.1\left(\mathrm{C}^{\mathrm{C} 2 \mathrm{~b}}\right)$, $123.5\left(\mathrm{C}^{\mathrm{A} 3}\right), 122.8\left(\mathrm{C}^{\mathrm{B} 3}\right), 120.5\left(\mathrm{C}^{\mathrm{C} 6 \mathrm{~b}}\right), 119.9\left(\mathrm{C}^{\mathrm{C} 6 \mathrm{a}}\right) .{ }^{31} \mathrm{P}$ NMR $\left(202 \mathrm{MHz}\right.$, acetone- $\left.d_{6}, \mathrm{~T}=253 \mathrm{~K}\right) \delta / \mathrm{ppm}-12.7$ (broad, FWHM $=200 \mathrm{~Hz}),-15.5(\mathrm{br}, \mathrm{FWHM}=220 \mathrm{~Hz}),-144.5$ (septet, $J_{\mathrm{PF}}=$ $\left.710 \mathrm{~Hz},\left[\mathrm{PF}_{6}\right]^{-}\right)$. ESI MS: $m / z 883.0\left[{\mathrm{M}-\mathrm{PF}_{6}}^{+}\right.$(base peak, calc.

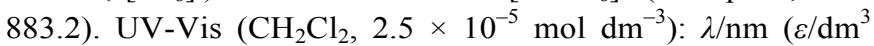
$\left.\mathrm{mol}^{-1} \mathrm{~cm}^{-1}\right) 230$ (57400), 287 (21900), 327sh (10500), 383 (2300). Found: $\mathrm{C}$ 64.94, $\mathrm{H} 4.49, \mathrm{~N} 3.05 ; \mathrm{C}_{56} \mathrm{H}_{42} \mathrm{CuF}_{6} \mathrm{~N}_{2} \mathrm{OP}_{3}$ requires $\mathrm{C} 65.34, \mathrm{H} 4.11, \mathrm{~N} 2.72 \%$.

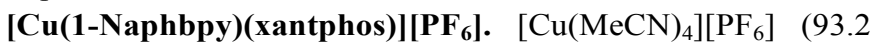
$\mathrm{mg}, 0.25 \mathrm{mmol})$ was dissolved in $\mathrm{CH}_{2} \mathrm{Cl}_{2}(15 \mathrm{ml})$. A solution of xantphos (148 mg, $0.25 \mathrm{mmol}$ ) and 1-Naphbpy (70.6 mg, 0.25 mmol) was added and the mixture turned red then yellow while 
it was stirred for $2 \mathrm{~h}$ at room temperature. The yellow solution was filtered and the solvent was removed from the filtrate. The solid material was washed with hexane $(2 \times 30 \mathrm{ml})$ and dried under vacuum. [Cu(1-Naphbpy)(xantphos)][PF 6 (251 mg, 0.24 mmol, 94\%) was isolated as a yellow solid. ${ }^{1} \mathrm{H}$ NMR (500 $\mathrm{MHz}$, acetone- $\left.d_{6}, 223 \mathrm{~K}\right) \delta / \mathrm{ppm}: 8.87(\mathrm{dd}, J=8.1,1.1 \mathrm{~Hz}, 1 \mathrm{H}$, $\left.\mathrm{H}^{\mathrm{B} 3}\right), 8.83\left(\mathrm{~d}, J=8.2 \mathrm{~Hz}, 1 \mathrm{H}, \mathrm{H}^{\mathrm{A} 3}\right), 8.41(\mathrm{t}, J=7.9 \mathrm{~Hz}, 1 \mathrm{H}$, $\left.\mathrm{H}^{\mathrm{B} 4}\right), 8.17\left(\mathrm{~d}, J=8.3 \mathrm{~Hz}, 1 \mathrm{H}, \mathrm{H}^{\mathrm{N} 5}\right), 8.30(\mathrm{~d}, J=8.3 \mathrm{~Hz}, 1 \mathrm{H}$, $\left.\mathrm{H}^{\mathrm{N} 4}\right), 8.14\left(\mathrm{td}, J=7.9,1.7 \mathrm{~Hz}, 1 \mathrm{H}, \mathrm{H}^{\mathrm{A} 4}\right), 7.84(\mathrm{dt}, J=8.0,1.6$ $\left.\mathrm{Hz}, 2 \mathrm{H}, \mathrm{H}^{\mathrm{C} 5 \mathrm{a}+\mathrm{C} 5 \mathrm{~b}}\right), 7.61\left(\mathrm{dd}, J=7.7,1.0 \mathrm{~Hz}, 1 \mathrm{H}, \mathrm{H}^{\mathrm{B} 5}\right), 7.57-7.49$ (overlapping $\left.\mathrm{m}, 2 \mathrm{H}, \mathrm{H}^{\mathrm{D} 4 \mathrm{a}+\mathrm{N} 6}\right), 7.46(\mathrm{td}, J=7.4,1.3 \mathrm{~Hz}, 1 \mathrm{H}$, $\mathrm{H}^{\mathrm{D} 4 \mathrm{~b}}$ ), 7.37-7.26 (overlapping $\mathrm{m}, 5 \mathrm{H}, \mathrm{H}^{\mathrm{D} 3 \mathrm{a}+\mathrm{D} 3 \mathrm{~b}+\mathrm{C} 4 \mathrm{a}}$ ), 7.25-7.13 (overlapping $\mathrm{m}, 4 \mathrm{H}, \mathrm{H}^{\mathrm{C} 4 \mathrm{~b}+\mathrm{N} 3+\mathrm{D} 4 \mathrm{c}+\mathrm{D} 4 \mathrm{~d}}$ ), 7.08-7.03 (overlapping $\mathrm{m}, 2 \mathrm{H}, \mathrm{H}^{\mathrm{A} 5+\mathrm{N} 8}$ ), 6.90-6.82 (overlapping $\mathrm{m}, 4 \mathrm{H}, \mathrm{H}^{\mathrm{D} 3 \mathrm{c}+\mathrm{D} 3 \mathrm{~d}}$ ), 6.696.53 (overlapping $\left.\mathrm{m}, 7 \mathrm{H}, \mathrm{H}^{\mathrm{A} 6+\mathrm{D} 2 \mathrm{a}+\mathrm{D} 2 \mathrm{~b}+\mathrm{C} 3 \mathrm{a}+\mathrm{N} 7}\right), 6.46(\mathrm{~d}, J=6.5$ $\mathrm{Hz}, 1 \mathrm{H}, \mathrm{H}^{\mathrm{N} 2}$ ), 6.30-6.20 (overlapping $\mathrm{m}, 3 \mathrm{H}, \mathrm{H}^{\mathrm{C} 3 \mathrm{~b}+\mathrm{D} 2 \mathrm{c}}$ ), 5.66 (broad $\left.\mathrm{m}, 2 \mathrm{H}, \mathrm{H}^{\mathrm{D} 2 \mathrm{~d}}\right), 2.06\left(\mathrm{~s}, \mathrm{H}^{\mathrm{Me}}\right.$, overlaps with acetone- $d_{6}$ and assigned from $2 \mathrm{D}$ spectra $), 1.32\left(\mathrm{~s}, 3 \mathrm{H}, \mathrm{H}^{\mathrm{Me}}\right) .{ }^{13} \mathrm{C} \mathrm{NMR}(126$ $\mathrm{MHz}$, acetone- $\left.d_{6}, 223 \mathrm{~K}\right) \delta / \mathrm{ppm}: 160.2\left(\mathrm{C}^{\mathrm{B} 6}\right), 155.0\left(\mathrm{C}^{\mathrm{Cla}}\right)$, $154.4\left(\mathrm{C}^{\mathrm{C} 1 \mathrm{~b}}\right), 153.5\left(\mathrm{C}^{\mathrm{A} 2}\right), 153.0\left(\mathrm{C}^{\mathrm{B} 2}\right), 147.5\left(\mathrm{C}^{\mathrm{A} 6}\right), 139.7$ $\left(\mathrm{C}^{\mathrm{A} 4+\mathrm{B} 4}\right), 139.0\left(\mathrm{C}^{\mathrm{N} 1}\right), 134.6\left(\mathrm{C}^{\mathrm{N} 4 \mathrm{a}}\right), 134.5\left(\mathrm{C}^{\mathrm{C} 6 \mathrm{a}+\mathrm{C} 6 \mathrm{~b}}\right), 134.4$ $\left(\mathrm{C}^{\mathrm{D} 2 \mathrm{a}}\right), 134.0\left(\mathrm{C}^{\mathrm{D} 2 \mathrm{c}}\right), 133.3\left(\mathrm{C}^{\mathrm{D} 2 \mathrm{~b}}\right), 132.4\left(\mathrm{C}^{\mathrm{D} 2 \mathrm{~d}}\right), 131.3\left(\mathrm{C}^{\mathrm{C} 3 \mathrm{~b}}\right)$, $131.2\left(\mathrm{C}^{\mathrm{D} 4 \mathrm{a}}\right), 131.1\left(\mathrm{C}^{\mathrm{N} 8 \mathrm{a}}\right), 130.9\left(\mathrm{C}^{\mathrm{C} 3 \mathrm{a}}\right), 130.7\left(\mathrm{C}^{\mathrm{D} 4 \mathrm{~b}}\right), 130.1$ $\left(\mathrm{C}^{\mathrm{D} 4 \mathrm{c}}\right), \quad 130.1 \quad\left(\mathrm{C}^{\mathrm{N} 4}\right), \quad 129.7 \quad\left(\mathrm{C}^{\mathrm{D} 4 \mathrm{~d}}\right), \quad 129.6 \quad\left(\mathrm{C}^{\mathrm{Dla}}\right), \quad 129.5$ $\left(\mathrm{C}^{\mathrm{D} 3 \mathrm{a}+\mathrm{D} 3 \mathrm{~b}}\right), 129.4\left(\mathrm{C}^{\mathrm{B} 5}\right), 129.3\left(\mathrm{C}^{\mathrm{N} 5}\right), 128.9\left(\mathrm{C}^{\mathrm{D} 1 \mathrm{c}+\mathrm{D} 1 \mathrm{~d}}\right), 128.7$ $\left(\mathrm{C}^{\mathrm{D} 3 \mathrm{c}+\mathrm{D} 3 \mathrm{~d}}\right), 128.0\left(\mathrm{C}^{\mathrm{C} 5 \mathrm{a}+\mathrm{C} 5 \mathrm{~b}}\right), 127.5\left(\mathrm{C}^{\mathrm{N} 7}\right), 127.4\left(\mathrm{C}^{\mathrm{N} 2}\right), 127.3$ $\left(\mathrm{C}^{\mathrm{N} 6}\right), 126.8\left(\mathrm{C}^{\mathrm{N} 3}\right), 126.1\left(\mathrm{C}^{\mathrm{C} 4 \mathrm{~b}}\right), 125.9\left(\mathrm{C}^{\mathrm{C} 4 \mathrm{a}}\right), 125.8\left(\mathrm{C}^{\mathrm{N} 8+\mathrm{A} 5}\right)$, $124.5\left(\mathrm{C}^{\mathrm{A} 3}\right), 123.3\left(\mathrm{C}^{\mathrm{B} 3}\right), 121.5\left(\mathrm{C}^{\mathrm{C} 2 \mathrm{a}}\right), 120.2\left(\mathrm{C}^{\mathrm{C} 2 \mathrm{~b}}\right), 36.7\left(\mathrm{C}^{\mathrm{q}}\right)$, $32.0\left(\mathrm{C}^{\mathrm{Me}}\right), 23.0\left(\mathrm{C}^{\mathrm{Me}}\right) .{ }^{31} \mathrm{P}$ NMR $\left(202 \mathrm{MHz}\right.$, acetone- $d_{6}, 223$ K) $\delta / \mathrm{ppm}-10.9($ broad d, $J=110 \mathrm{~Hz}),-13.4($ broad d, $J=110$ $\mathrm{Hz}$ ), -144.5 (septet, $J_{\mathrm{PF}}=710 \mathrm{~Hz},\left[\mathrm{PF}_{6}\right]^{-}$). ESI MS: $m / z 923.1$ $\left[\mathrm{M}-\mathrm{PF}_{6}\right]^{+}$(base peak, calc. 923.2). UV-Vis $\left(\mathrm{CH}_{2} \mathrm{Cl}_{2}, 2.5 \times 10^{-5}\right.$ $\left.\mathrm{mol} \mathrm{dm}{ }^{-3}\right): \lambda / \mathrm{nm}\left(\varepsilon / \mathrm{dm}^{3} \mathrm{~mol}^{-1} \mathrm{~cm}^{-1}\right) 230$ (60800), 289 (29900), 328sh (7800), 392 (2500). Found: C 66.29, H 4.78, N 2.86; $\mathrm{C}_{59} \mathrm{H}_{46} \mathrm{CuF}_{6} \mathrm{~N}_{2} \mathrm{OP}_{3}$ requires $\mathrm{C} 66.26, \mathrm{H} 4.34, \mathrm{~N} 2.62 \%$.

[Cu(2-Naphbpy)(POP)][PF 6 . $\quad$ [Cu(2-Naphbpy)(POP)][PF 6$]$ was prepared according to the procedure for $[\mathrm{Cu}(1-$ Naphbpy)(POP)][PF $]$ using 2-Naphbpy (70.6 mg, $0.25 \mathrm{mmol})$ in place of 1 -Naphbpy. [Cu(2-Naphbpy)(POP) $]\left[\mathrm{PF}_{6}\right]$ was isolated as a yellow powder $(251 \mathrm{mg}, 0.24 \mathrm{mmol}, 98 \%) .{ }^{1} \mathrm{H}$ NMR $\left(500 \mathrm{MHz}\right.$, acetone- $d_{6}$ ) $\delta / \mathrm{ppm}$ : 8.67-8.62 (overlapping m, $\left.2 \mathrm{H}, \mathrm{H}^{\mathrm{A} 3+\mathrm{B} 3}\right), 8.30\left(\mathrm{t}, J=7.9 \mathrm{~Hz}, 1 \mathrm{H}, \mathrm{H}^{\mathrm{B} 4}\right), 8.12(\mathrm{~d}, J=5.1 \mathrm{~Hz}$, $\left.1 \mathrm{H}, \mathrm{H}^{\mathrm{A} 6}\right), 8.09\left(\mathrm{td}, J=7.9,1.7 \mathrm{~Hz}, 1 \mathrm{H}, \mathrm{H}^{\mathrm{A} 4}\right), 7.91\left(\mathrm{~m}, 1 \mathrm{H}, \mathrm{H}^{\mathrm{N} 5}\right)$, $7.76\left(\mathrm{dd}, J=7.7,1.0 \mathrm{~Hz}, 1 \mathrm{H}, \mathrm{H}^{\mathrm{B} 5}\right.$ ), 7.65-7.61 (overlapping $\mathrm{m}$, $2 \mathrm{H}, \mathrm{H}^{\mathrm{N} 6+\mathrm{N} 8}$ ), 7.57-7.52 (overlapping $\mathrm{m}, 2 \mathrm{H}, \mathrm{H}^{\mathrm{N} 1+\mathrm{N} 7}$ ), 7.44-7.33 (overlapping $\left.\mathrm{m}, 6 \mathrm{H}, \mathrm{H}^{\mathrm{N} 3+\mathrm{C} 5+\mathrm{D} 4 \mathrm{a}+\mathrm{N} 4}\right), 7.31\left(\mathrm{~m}, 2 \mathrm{H}, \mathrm{H}^{\mathrm{D} 4 \mathrm{~b}}\right), 7.25$ $\left(\mathrm{m}, 4 \mathrm{H}, \mathrm{H}^{\mathrm{D3a}}\right), 7.16\left(\mathrm{~m}, 2 \mathrm{H}, \mathrm{H}^{\mathrm{C} 6}\right), 7.13(\mathrm{ddd}, J=7.6,5.2,1.1$ $\mathrm{Hz}, 1 \mathrm{H}, \mathrm{H}^{\mathrm{A} 5}$ ), 7.05-6.95 (overlapping $\left.\mathrm{m}, 6 \mathrm{H}, \mathrm{H}^{\mathrm{C} 4+\mathrm{D} 3 \mathrm{~b}}\right), 6.83(\mathrm{~m}$, $4 \mathrm{H}, \mathrm{H}^{\mathrm{D} 2 \mathrm{a}}$ ), 6.64-6.54 (overlapping $\mathrm{m}, 6 \mathrm{H}, \mathrm{H}^{\mathrm{C} 3+\mathrm{D} 2 \mathrm{~b}}$ ). ${ }^{13} \mathrm{C} \mathrm{NMR}$ $\left(126 \mathrm{MHz}\right.$, acetone- $\left.d_{6}\right) \delta / \mathrm{ppm}: 161.8\left(\mathrm{C}^{\mathrm{B} 6}\right), 158.2(\mathrm{t}, J=5.9 \mathrm{~Hz}$, $\left.\mathrm{C}^{\mathrm{C} 1}\right), 154.4\left(\mathrm{C}^{\mathrm{B} 2}\right), 153.8\left(\mathrm{C}^{\mathrm{A} 2}\right), 149.7\left(\mathrm{C}^{\mathrm{A} 6}\right), 140.3\left(\mathrm{C}^{\mathrm{B} 4}\right), 139.7$ $\left(\mathrm{C}^{\mathrm{A} 4}\right), 139.0\left(\mathrm{C}^{\mathrm{N} 2}\right), 135.3\left(\mathrm{C}^{\mathrm{C} 3}\right), 134.6\left(\mathrm{C}^{\mathrm{D} 2 \mathrm{~b}}\right), 134.4\left(\mathrm{C}^{\mathrm{N} 4 \mathrm{a}}\right)$, $133.5\left(\mathrm{C}^{\mathrm{N} 8 \mathrm{a}+\mathrm{D} 2 \mathrm{a}}\right), 133.1\left(\mathrm{C}^{\mathrm{C} 5}\right), 131.0\left(\mathrm{C}^{\mathrm{D} 4 \mathrm{a}}\right), 130.8\left(\mathrm{C}^{\mathrm{D} 4 \mathrm{~b}}\right), 130.1$ $\left(\mathrm{C}^{\mathrm{N} 4}\right), 129.7\left(\mathrm{C}^{\mathrm{D} 3 \mathrm{a}}\right), 129.5\left(\mathrm{C}^{\mathrm{N} 8}\right), 129.3\left(\mathrm{C}^{\mathrm{D} 3 \mathrm{~b}}\right), 128.8\left(\mathrm{C}^{\mathrm{N} 1+\mathrm{N} 5}\right)$, $128.1\left(\mathrm{C}^{\mathrm{N} 6}\right), 128.0\left(\mathrm{C}^{\mathrm{B} 5}\right), 127.9\left(\mathrm{C}^{\mathrm{N} 7}\right), 126.4\left(\mathrm{C}^{\mathrm{A} 5+\mathrm{N} 3}\right), 125.9(\mathrm{t}$, $\left.J=2.1 \mathrm{~Hz}, \mathrm{C}^{\mathrm{C} 4}\right), 124.6\left(\mathrm{t}, J=14.2 \mathrm{~Hz}, \mathrm{C}^{\mathrm{C} 2}\right), 124.0\left(\mathrm{C}^{\mathrm{A} 3}\right), 123.0$
$\left(\mathrm{C}^{\mathrm{B} 3}\right), 120.6\left(\mathrm{C}^{\mathrm{C} 6}\right) .{ }^{31} \mathrm{P}$ NMR $\left(202 \mathrm{MHz}\right.$, acetone- $\left.d_{6}\right) \delta / \mathrm{ppm}-$ 13.4 (broad, FWHM $=200 \mathrm{~Hz}),-144.3\left(\right.$ septet, $J_{\mathrm{PF}}=710 \mathrm{~Hz}$, $\left[\mathrm{PF}_{6}\right]^{-}$). ESI MS: $m / z$ 883.1 [M-PF $]^{+}$(base peak, calc. 883.2). UV-Vis $\left(\mathrm{CH}_{2} \mathrm{Cl}_{2}, 2.5 \times 10^{-5} \mathrm{~mol} \mathrm{dm}{ }^{-3}\right): \lambda / \mathrm{nm}\left(\varepsilon / \mathrm{dm}^{3} \mathrm{~mol}^{-1} \mathrm{~cm}^{-}\right.$ 1) 232 (67600), 288 (28800), 389 (2600). Found: C 64.94, H 4.61, $\mathrm{N} 2.92 ; \mathrm{C}_{56} \mathrm{H}_{42} \mathrm{CuF}_{6} \mathrm{~N}_{2} \mathrm{OP}_{3}$ requires $\mathrm{C} 65.34, \mathrm{H} \mathrm{4.11,} \mathrm{N}$ $2.72 \%$.

[Cu(2-Naphbpy)(xantphos)][PF $\mathbf{6}]$. This compound was prepared by the same procedure as for $[\mathrm{Cu}(1-$ Naphbpy)(xantphos)][PF 6 using 2-Naphbpy (70.6 mg, 0.25

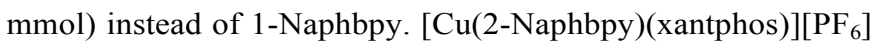
was isolated as a yellow powder $(256 \mathrm{mg}, 0.24 \mathrm{mmol}, 96 \%) .{ }^{1} \mathrm{H}$ NMR (500 MHz, acetone- $\left.d_{6}\right) \delta / \mathrm{ppm}: 8.63(\mathrm{dt}, J=8.3,1.0 \mathrm{~Hz}$, $\left.1 \mathrm{H}, \mathrm{H}^{\mathrm{A} 3}\right), 8.55\left(\mathrm{dd}, J=8.0,1.0 \mathrm{~Hz}, 1 \mathrm{H}, \mathrm{H}^{\mathrm{B} 3}\right), 8.24(\mathrm{t}, J=7.9$ $\left.\mathrm{Hz}, 1 \mathrm{H}, \mathrm{H}^{\mathrm{B} 4}\right), 8.12\left(\mathrm{~m} 1 \mathrm{H}, \mathrm{H}^{\mathrm{A} 4}\right), 8.00\left(\mathrm{~m}, 1 \mathrm{H}, \mathrm{H}^{\mathrm{N} 5}\right), 7.77-7.73$ (overlapping $\mathrm{m}, 4 \mathrm{H}, \mathrm{H}^{\mathrm{C} 5+\mathrm{B} 5+\mathrm{N} 1}$ ), 7.67 (ddd, $J=8.3,6.8,1.3 \mathrm{~Hz}$, $\left.1 \mathrm{H}, \mathrm{H}^{\mathrm{N} 6}\right), 7.53\left(\mathrm{ddd}, J=8.1,6.7,1.2 \mathrm{~Hz}, 1 \mathrm{H}, \mathrm{H}^{\mathrm{N} 7}\right), 7.50-7.42$ (overlapping $\mathrm{m} \mathrm{Hz}, 4 \mathrm{H}, \mathrm{H}^{\mathrm{N} 3+\mathrm{N} 8+\mathrm{D} 4 \mathrm{a}}$ ), $7.28(\mathrm{t}, J=7.7 \mathrm{~Hz}, 4 \mathrm{H}$, $\mathrm{H}^{\mathrm{D} 3 \mathrm{a}}$ ), $7.28\left(\mathrm{t}, J=7.7 \mathrm{~Hz}, 2 \mathrm{H}, \mathrm{H}^{\mathrm{C} 4}\right), 7.17-7.11$ (overlapping $\mathrm{m}$, $\left.3 \mathrm{H}, \mathrm{H}^{\mathrm{A} 5+\mathrm{D} 4 \mathrm{~b}}\right), 6.82\left(\mathrm{~m} \mathrm{~Hz}, 4 \mathrm{H}, \mathrm{H}^{\mathrm{D} 2 \mathrm{a}}\right), 6.80-6.73$ (overlapping $\mathrm{m}$, $5 \mathrm{H}, \mathrm{H}^{\mathrm{A} 6+\mathrm{D} 3 \mathrm{~b}}$ ), 6.67-6.59 (overlapping $\mathrm{m}, 6 \mathrm{H}, \mathrm{H}^{\mathrm{C} 3+\mathrm{D} 2 \mathrm{~b}}$ ), 1.91 (broad s, 3H, He $\left.{ }^{\mathrm{Me}}\right), 1.46\left(\mathrm{~s}, 3 \mathrm{H}, \mathrm{H}^{\mathrm{Me}}\right) .{ }^{13} \mathrm{C}$ NMR $(126 \mathrm{MHz}$, acetone- $\left.d_{6}\right) \delta / \mathrm{ppm}$ : $161.7\left(\mathrm{C}^{\mathrm{B} 6}\right), 155.5\left(\mathrm{t}, J=6.3 \mathrm{~Hz}, \mathrm{C}^{\mathrm{C} 1}\right)$, $154.3\left(\mathrm{C}^{\mathrm{A} 2}\right), 154.0\left(\mathrm{C}^{\mathrm{B} 2}\right), 148.9\left(\mathrm{C}^{\mathrm{A} 6}\right), 140.3\left(\mathrm{C}^{\mathrm{B} 4}\right), 140.1\left(\mathrm{C}^{\mathrm{A} 4}\right)$, $139.2\left(\mathrm{C}^{\mathrm{N} 2}\right), 135.6\left(\mathrm{C}^{\mathrm{D} 1 \mathrm{a}}\right), 134.9\left(\mathrm{C}^{\mathrm{C} 6}\right), 134.5\left(\mathrm{C}^{\mathrm{N} 4 \mathrm{a}}\right), 134.1$ $\left(\mathrm{C}^{\mathrm{N} 8 \mathrm{a}}\right), 133.9\left(\mathrm{t}, J=7.7 \mathrm{~Hz}, \mathrm{C}^{\mathrm{D} 2 \mathrm{a}+\mathrm{D} 2 \mathrm{~b}}\right), 131.5\left(\mathrm{C}^{\mathrm{C} 3}\right), 131.1\left(\mathrm{C}^{\mathrm{D} 4 \mathrm{a}}\right)$, $130.8\left(\mathrm{C}^{\mathrm{D} 1 \mathrm{~b}}\right), 130.6\left(\mathrm{C}^{\mathrm{D} 4 \mathrm{~b}}\right), 129.8\left(\mathrm{t}, J=4.5 \mathrm{~Hz}, \mathrm{C}^{\mathrm{D} 3 \mathrm{a}}\right), 129.5$ $\left(\mathrm{C}^{\mathrm{N} 8}\right), 129.1\left(\mathrm{t}, J=4.7 \mathrm{~Hz}, \mathrm{C}^{\mathrm{D} 3 \mathrm{a}}\right), 128.9\left(\mathrm{C}^{\mathrm{N} 5}\right), 128.7\left(\mathrm{C}^{\mathrm{N} 1}\right)$, $128.3\left(\mathrm{C}^{\mathrm{C} 5}\right), 128.2\left(\mathrm{C}^{\mathrm{N} 6}\right), 127.9\left(\mathrm{C}^{\mathrm{B} 5}\right), 126.4\left(\mathrm{C}^{\mathrm{A} 5}\right), 126.2(\mathrm{t}, J=$ $\left.2.2 \mathrm{~Hz}, \mathrm{C}^{\mathrm{C} 4}\right), 126.0\left(\mathrm{C}^{\mathrm{N} 3}\right), 124.5\left(\mathrm{C}^{\mathrm{A} 3}\right), 123.3\left(\mathrm{C}^{\mathrm{B} 3}\right), 121.9$ $\left(\mathrm{C}^{\mathrm{N} 7}\right), 121.1\left(\mathrm{t}, J=13.1 \mathrm{~Hz}, \mathrm{C}^{\mathrm{C} 2}\right), 36.8\left(\mathrm{C}^{\mathrm{q}}\right), 30.8\left(\mathrm{C}^{\mathrm{Me}}\right), 25.3$ $\left(\mathrm{C}^{\mathrm{Me}}\right) .{ }^{31} \mathrm{P}$ NMR $\left(202 \mathrm{MHz}\right.$, acetone- $\left.d_{6}\right) \delta / \mathrm{ppm}-12.8$ (broad, $\mathrm{FWHM}=210 \mathrm{~Hz}$ ), -144.3 (septet, $J_{\mathrm{PF}}=710 \mathrm{~Hz},\left[\mathrm{PF}_{6}\right]^{-}$). ESI MS: $m / z$ 923.2 $\left[\mathrm{M}-\mathrm{PF}_{6}\right]^{+}$(base peak, calc. 923.2). UV-Vis $\left(\mathrm{CH}_{2} \mathrm{Cl}_{2}, 2.5 \times 10^{-5} \mathrm{~mol} \mathrm{dm}{ }^{-3}\right): \lambda / \mathrm{nm}\left(\varepsilon / \mathrm{dm}^{3} \mathrm{~mol}^{-1} \mathrm{~cm}^{-1}\right) 230$ (67000), 284 (32800), 388 (2500). Found: C 66.18, H 4.79, N 2.90; $\mathrm{C}_{59} \mathrm{H}_{46} \mathrm{CuF}_{6} \mathrm{~N}_{2} \mathrm{OP}_{3}$ requires $\mathrm{C} 66.26, \mathrm{H} 4.34, \mathrm{~N} 2.62 \%$.

[Cu(1-Pyrbpy)(POP)] $\left[\mathbf{P F}_{6}\right]$. [Cu(MeCN $\left.)_{4}\right]\left[\mathrm{PF}_{6}\right] \quad(93.2 \mathrm{mg}$, $0.25 \mathrm{mmol})$ and POP (148 $\mathrm{mg}, 0.27 \mathrm{mmol})$ were dissolved in $\mathrm{CH}_{2} \mathrm{Cl}_{2}(30 \mathrm{ml})$ and the mixture was stirred for $2 \mathrm{~h}$ at room temperature. 1-Pyrbpy $(89.6 \mathrm{mg}, 0.25 \mathrm{mmol})$ was added and stirring was continued for $2 \mathrm{~h}$. The yellow solution was filtered and the solvent was removed from the filtrate. The solid material was washed with hexane $(2 \times 30 \mathrm{ml})$, dried under vacuum and redissolved in a small amount of acetone. The solution was layered with $\mathrm{Et}_{2} \mathrm{O}$ and left to crystallize for 2 days. The resulting yellow crystals were ground to a powder and dried under vacuum to give $[\mathrm{Cu}(1-\mathrm{Pyrbpy})(\mathrm{POP})]\left[\mathrm{PF}_{6}\right](105$ $\mathrm{mg}, 0.10 \mathrm{mmol}, 38 \%)$ as a yellow powder. ${ }^{1} \mathrm{H} \mathrm{NMR}(600 \mathrm{MHz}$, acetone- $\left.d_{6}, 238 \mathrm{~K}\right) \delta / \mathrm{ppm}: 8.89\left(\mathrm{dd}, J=8.2,1.0 \mathrm{~Hz}, 1 \mathrm{H}, \mathrm{H}^{\mathrm{B} 3}\right)$, $8.78\left(\mathrm{~d}, J=8.3 \mathrm{~Hz}, 1 \mathrm{H}, \mathrm{H}^{\mathrm{A} 3}\right), 8.47\left(\mathrm{~d}, J=7.6 \mathrm{~Hz}, 1 \mathrm{H}, \mathrm{H}^{\mathrm{P} 6}\right)$, $8.45\left(\mathrm{t}, J=7.8 \mathrm{~Hz}, 1 \mathrm{H}, \mathrm{H}^{\mathrm{B} 4}\right), 8.42\left(\mathrm{~d}, J=8.9 \mathrm{~Hz}, 1 \mathrm{H}, \mathrm{H}^{\mathrm{P} 5}\right), 8.32$ $\left(\mathrm{d}, J=5.1 \mathrm{~Hz}, 1 \mathrm{H}, \mathrm{H}^{\mathrm{A6}}\right), 8.23\left(\mathrm{~d}, J=7.6 \mathrm{~Hz}, 1 \mathrm{H}, \mathrm{H}^{\mathrm{P} 8}\right), 8.19(\mathrm{~d}$, $\left.J=8.9 \mathrm{~Hz}, 1 \mathrm{H}, \mathrm{H}^{\mathrm{P} 4}\right), 8.14\left(\mathrm{t}, J=7.6 \mathrm{~Hz}, 1 \mathrm{H}, \mathrm{H}^{\mathrm{P} 7}\right), 8.13(\mathrm{t}, J=$ $\left.8.3 \mathrm{~Hz}, 1 \mathrm{H}, \mathrm{H}^{\mathrm{A} 4}\right), 7.80\left(\mathrm{dd}, J=7.6,1.0 \mathrm{~Hz}, 1 \mathrm{H}, \mathrm{H}^{\mathrm{B} 5}\right), 7.70(\mathrm{~d}, J$ $\left.=7.2 \mathrm{~Hz}, 1 \mathrm{H}, \mathrm{H}^{\mathrm{P} 2}\right), 7.69\left(\mathrm{~d}, J=9.4 \mathrm{~Hz}, 1 \mathrm{H}, \mathrm{H}^{\mathrm{P} 9}\right), 7.62(\mathrm{t}, J=$ 
$\left.7.5 \mathrm{~Hz}, 1 \mathrm{H}, \mathrm{H}^{\mathrm{D} 4 \mathrm{a}}\right), 7.53\left(\mathrm{t}, J=7.5 \mathrm{~Hz}, 1 \mathrm{H}, \mathrm{H}^{\mathrm{D} 4 \mathrm{~b}}\right), 7.45-7.42$ (overlapping $\mathrm{m} \mathrm{Hz}, 2 \mathrm{H}, \mathrm{H}^{\mathrm{D} 4 \mathrm{c}+\mathrm{C} 5 \mathrm{~b}}$ ), 7.38-7.30 (overlapping $\mathrm{m}$, $\left.10 \mathrm{H}, \mathrm{H}^{\mathrm{C} 6 \mathrm{a}+\mathrm{C} 6 \mathrm{~b}+\mathrm{C} 5 \mathrm{a}+\mathrm{D} 3 \mathrm{a}+\mathrm{D} 3 \mathrm{~b}+\mathrm{D} 3 \mathrm{c}+\mathrm{P} 3}\right), 7.16(\mathrm{ddd}, J=7.6,5.1,1.1 \mathrm{~Hz}$, $1 \mathrm{H}, \mathrm{H}^{\mathrm{A} 5}$ ), $7.09\left(\mathrm{t}, J=8.2 \mathrm{~Hz}, 2 \mathrm{H}, \mathrm{H}^{\mathrm{D} 2 \mathrm{c}}\right.$ ), 7.04-7.01 (overlapping $\left.\mathrm{m}, 2 \mathrm{H}, \mathrm{H}^{\mathrm{C} 4 \mathrm{~b}+\mathrm{P} 10}\right), 6.95\left(\mathrm{t}, J=7.7 \mathrm{~Hz}, 1 \mathrm{H}, \mathrm{H}^{\mathrm{C} 4 \mathrm{a}}\right), 6.70(\mathrm{t}, J=7.7$ $\mathrm{Hz}, 1 \mathrm{H}, \mathrm{H}^{\mathrm{D} 4 \mathrm{~d}}$ ), 6.66 (ddd, $\left.J=7.8,6.2,1.7 \mathrm{~Hz}, 1 \mathrm{H}, \mathrm{H}^{\mathrm{C} 3 \mathrm{~b}}\right), 6.52$ $\left(\mathrm{t}, J=8.1 \mathrm{~Hz}, 2 \mathrm{H}, \mathrm{H}^{\mathrm{D} 2 \mathrm{a}}\right), 6.39\left(\mathrm{t}, J=8.2 \mathrm{~Hz}, 2 \mathrm{H}, \mathrm{H}^{\mathrm{D} 2 \mathrm{~b}}\right), 6.13$ $\left(\mathrm{dd}, J=7.8,6.2,1.7 \mathrm{~Hz}, 1 \mathrm{H}, \mathrm{H}^{\mathrm{C} 3 \mathrm{a}}\right), 6.03\left(\mathrm{~m} \mathrm{~Hz}, 2 \mathrm{H}, \mathrm{H}^{\mathrm{D} 3 \mathrm{~d}}\right), 5.63$ $\left(\mathrm{m} \mathrm{Hz}, 2 \mathrm{H}, \mathrm{H}^{\mathrm{D} 2 \mathrm{~d}}\right) .{ }^{13} \mathrm{C}$ NMR $\left(151 \mathrm{MHz}\right.$, acetone- $\left.d_{6}, 238 \mathrm{~K}\right)$ $\delta /$ ppm: $159.8\left(\mathrm{C}^{\mathrm{B} 6}\right), 158.3\left(\mathrm{C}^{\mathrm{Clb}}\right), 158.3\left(\mathrm{C}^{\mathrm{Clb}}\right), 154.3\left(\mathrm{C}^{\mathrm{B} 3}\right)$, $152.9\left(\mathrm{C}^{\mathrm{A} 2}\right), 149.5\left(\mathrm{C}^{\mathrm{A} 6}\right), 139.7\left(\mathrm{C}^{\mathrm{A} 4}\right), 139.2\left(\mathrm{C}^{\mathrm{B} 4}\right), 136.0$ $\left(\mathrm{C}^{\mathrm{C} 3 \mathrm{~b}}\right), 135.5\left(\mathrm{C}^{\mathrm{P} 1}\right), 134.7\left(\mathrm{C}^{\mathrm{C} 3 \mathrm{a}}\right), 133.7\left(\mathrm{C}^{\mathrm{D} 2 \mathrm{a}}\right), 133.4\left(\mathrm{C}^{\mathrm{D} 2 \mathrm{c}}\right)$, $133.1\left(\mathrm{C}^{\mathrm{D} 2 \mathrm{~b}}\right), 132.8\left(\mathrm{C}^{\mathrm{C} 5 \mathrm{~b}}\right), 132.7\left(\mathrm{C}^{\mathrm{D} 2 \mathrm{~d}}\right), 132.4\left(\mathrm{C}^{\mathrm{C} 5 \mathrm{a}}\right), 131.8$ $\left(\mathrm{C}^{\mathrm{P3a}}\right), 131.6\left(\mathrm{C}^{\mathrm{P} 5 \mathrm{a}}\right), 130.9\left(\mathrm{C}^{\mathrm{P8a}}\right), 130.5\left(\mathrm{C}^{\mathrm{D} 4 \mathrm{~b}+\mathrm{D} 4 \mathrm{c}}\right), 130.4\left(\mathrm{C}^{\mathrm{D} 4 \mathrm{a}}\right)$, $129.8\left(\mathrm{C}^{\mathrm{B} 5+\mathrm{D} 4 \mathrm{~d}}\right), 129.4\left(\mathrm{C}^{\mathrm{D} 3 \mathrm{~b}}\right), 129.3\left(\mathrm{C}^{\mathrm{D} 3 \mathrm{c}}\right), 129.2\left(\mathrm{C}^{\mathrm{P} 9}\right), 129.1$ $\left(\mathrm{C}^{\mathrm{D} 3 \mathrm{a}}\right), 129.0\left(\mathrm{C}^{\mathrm{P} 5}\right), 128.4\left(\mathrm{C}^{\mathrm{P} 10 \mathrm{a}}\right), 128.0\left(\mathrm{C}^{\mathrm{D} 3 \mathrm{~d}}\right), 127.9\left(\mathrm{C}^{\mathrm{P} 4}\right)$, $127.5\left(\mathrm{C}^{\mathrm{P} 2}\right), 127.1\left(\mathrm{C}^{\mathrm{P} 7}\right), 126.4\left(\mathrm{C}^{\mathrm{P} 6}\right), 126.1\left(\mathrm{C}^{\mathrm{P} 8+\mathrm{C} 4 \mathrm{a}}\right), 125.9$ $\left(\mathrm{C}^{\mathrm{A} 5}\right), 125.7\left(\mathrm{C}^{\mathrm{P} 3}\right), 124.7\left(\mathrm{C}^{\mathrm{C} 4 \mathrm{~b}+\mathrm{P} 10 \mathrm{~b}}\right), 124.5\left(\mathrm{C}^{\mathrm{P} 10 \mathrm{c}}\right), 123.6\left(\mathrm{C}^{\mathrm{A} 3}\right)$, $123.5\left(\mathrm{C}^{\mathrm{P} 10}\right), 122.2\left(\mathrm{C}^{\mathrm{C} 6 \mathrm{a}}\right), 117.7\left(\mathrm{C}^{\mathrm{C} 6 \mathrm{~b}}\right), 112.8\left(\mathrm{C}^{\mathrm{B} 3}\right) .{ }^{31} \mathrm{P} \mathrm{NMR}$ $\left(162 \mathrm{MHz}\right.$, acetone- $\left.d_{6}, 295 \mathrm{~K}\right) \delta / \mathrm{ppm}-13.7$ (broad, FWHM = $\sim 350 \mathrm{~Hz}$ ), -144.3 (septet, $\left.J_{\mathrm{PF}}=707 \mathrm{~Hz},\left[\mathrm{PF}_{6}\right]^{-}\right) ;(243 \mathrm{MHz}$, acetone- $\left.d_{6}, 238 \mathrm{~K}\right) \delta / \mathrm{ppm}-14.5$ (broad, FWHM $\left.=130 \mathrm{~Hz}\right)$. ESI MS: $m / z$ 957.2 $\left[\mathrm{M}-\mathrm{PF}_{6}\right]^{+}$(base peak, calc. 957.2). UV-Vis $\left(\mathrm{CH}_{2} \mathrm{Cl}_{2}, 2.5 \times 10^{-5} \mathrm{~mol} \mathrm{dm}{ }^{-3}\right): \lambda / \mathrm{nm}\left(\varepsilon / \mathrm{dm}^{3} \mathrm{~mol}^{-1} \mathrm{~cm}^{-1}\right) 230$ (58800), 245 (62000), 282 (38000), 354 (22500). Found: C $66.72, \mathrm{H} 4.47, \mathrm{~N} 2.60 ; \mathrm{C}_{62} \mathrm{H}_{44} \mathrm{CuF}_{6} \mathrm{~N}_{2} \mathrm{OP}_{3}$ requires $\mathrm{C} 67.48, \mathrm{H}$ $4.02, \mathrm{~N} 2.54 \%$.

[Cu(1-Pyrbpy)(xantphos)][PF 6 ] $\left[\mathrm{Cu}(\mathrm{MeCN})_{4}\right]\left[\mathrm{PF}_{6}\right](93.2 \mathrm{mg}$, $0.25 \mathrm{mmol})$ was dissolved in $\mathrm{CH}_{2} \mathrm{Cl}_{2}(15 \mathrm{ml})$. A solution of xantphos (148 mg, $0.25 \mathrm{mmol})$ and 1-Pyrbpy (89.6 mg, 0.25 mmol) was added and the mixture turned red then yellow while it was stirred for $2 \mathrm{~h}$ at room temperature. The yellow solution was filtered and the solvent was removed from the filtrate. The solid material was washed with hexane $(2 \times 30 \mathrm{ml})$ dried under vacuum and redissolved in a small amount of acetone. The solution was layered with $\mathrm{Et}_{2} \mathrm{O}$ and left to crystalize for 2 days. The resulting yellow crystals were ground to a powder and dried under vacuum to give $[\mathrm{Cu}(1-\mathrm{Pyrbpy})(\mathrm{xantphos})]\left[\mathrm{PF}_{6}\right]$
(115 mg, $0.10 \mathrm{mmol}, 40 \%)$ as a yellow powder. ${ }^{1} \mathrm{H}$ NMR (600 MHz, acetone- $\left.d_{6}, 238 \mathrm{~K}\right) \delta / \mathrm{ppm}: 8.89(\mathrm{dd}, J=8.2,1.1 \mathrm{~Hz}, 1 \mathrm{H}$, $\left.\mathrm{H}^{\mathrm{B} 3}\right), 8.87\left(\mathrm{~d}, J=8.2 \mathrm{~Hz}, 1 \mathrm{H}, \mathrm{H}^{\mathrm{A} 3}\right), 8.61(\mathrm{~d}, J=7.6 \mathrm{~Hz}, 1 \mathrm{H}$, $\left.\mathrm{H}^{\mathrm{P} 6}\right), 8.55\left(\mathrm{~d}, J=9.0 \mathrm{~Hz}, 1 \mathrm{H}, \mathrm{H}^{\mathrm{P} 5}\right), 8.50(\mathrm{~d}, J=7.8 \mathrm{~Hz}, 1 \mathrm{H}$, $\left.\mathrm{H}^{\mathrm{B} 4}\right), 8.34\left(\mathrm{~d}, J=9.0 \mathrm{~Hz}, 1 \mathrm{H}, \mathrm{H}^{\mathrm{P} 4}\right), 8.21(\mathrm{t}, J=7.6 \mathrm{~Hz}, 1 \mathrm{H}$, $\left.\mathrm{H}^{\mathrm{P} 7}\right), 8.16\left(\mathrm{td}, J=8.1,1.1 \mathrm{~Hz}, 1 \mathrm{H}, \mathrm{H}^{\mathrm{A} 4}\right), 8.14(\mathrm{~d}, J=7.6 \mathrm{~Hz}$, $\left.1 \mathrm{H}, \mathrm{H}^{\mathrm{P} 8}\right), 7.95\left(\mathrm{~d}, J=7.8 \mathrm{~Hz}, 1 \mathrm{H}, \mathrm{H}^{\mathrm{P} 3}\right), 7.82(\mathrm{dd}, J=7.9,1.4$ $\left.\mathrm{Hz}, 2 \mathrm{H}, \mathrm{H}^{\mathrm{C} 5 \mathrm{a}+\mathrm{C} 5 \mathrm{~b}}\right), 7.79\left(\mathrm{dd}, J=7.5,1.0 \mathrm{~Hz}, 1 \mathrm{H}, \mathrm{H}^{\mathrm{B} 5}\right), 7.66(\mathrm{~m}$, $\left.1 \mathrm{H}, \mathrm{H}^{\mathrm{D} 4 \mathrm{~d}}\right), 7.53\left(\mathrm{~m}, 1 \mathrm{H}, \mathrm{H}^{\mathrm{D} 4}\right), 7.43\left(\mathrm{~m}, 2 \mathrm{H}, \mathrm{H}^{\mathrm{D} 3 \mathrm{~d}}\right), 7.38(\mathrm{~m}, 2 \mathrm{H}$, $\left.\mathrm{H}^{\mathrm{D} 3 \mathrm{a}}\right), 7.37\left(\mathrm{~d}, J=9.2 \mathrm{~Hz}, 1 \mathrm{H}, \mathrm{H}^{\mathrm{P} 10}\right), 7.27(\mathrm{td}, J=7.8,1.1 \mathrm{~Hz}$, $\left.1 \mathrm{H}, \mathrm{H}^{\mathrm{Ca}}\right), 7.20\left(\mathrm{td}, J=7.8,1.2 \mathrm{~Hz}, 1 \mathrm{H}, \mathrm{H}^{\mathrm{C} 4 \mathrm{~b}}\right), 7.17(\mathrm{dd}, J=9.2$ $\left.\mathrm{Hz}, 1 \mathrm{H}, \mathrm{H}^{\mathrm{P} 9}\right), 7.04\left(\mathrm{ddd}, J=7.6,5.1,1.1 \mathrm{~Hz}, 1 \mathrm{H}, \mathrm{H}^{\mathrm{A} 5}\right), 7.00(\mathrm{~m}$, $\left.1 \mathrm{H}, \mathrm{H}^{\mathrm{D} 4 \mathrm{~b}}\right), 6.91\left(\mathrm{~d}, J=7.7 \mathrm{~Hz}, 1 \mathrm{H}, \mathrm{H}^{\mathrm{P} 2}\right), 6.71\left(\mathrm{~m}, 2 \mathrm{H}, \mathrm{H}^{\mathrm{D} 2 \mathrm{a}}\right)$, $6.65\left(\mathrm{~d}, J=5.1 \mathrm{~Hz}, 1 \mathrm{H}, \mathrm{H}^{\mathrm{A} 6}\right), 6.64-6.58$ (overlapping $\mathrm{m}, 3 \mathrm{H}$, $\left.\mathrm{H}^{\mathrm{D} 4 \mathrm{~b}+\mathrm{D} 2 \mathrm{~d}}\right), 6.53\left(\mathrm{~m}, 1 \mathrm{H}, \mathrm{H}^{\mathrm{C} 3 \mathrm{a}}\right), 6.43\left(\mathrm{t}, J=7.0 \mathrm{~Hz}, 2 \mathrm{H}, \mathrm{H}^{\mathrm{D} 3 \mathrm{c}}\right)$, $6.21\left(\mathrm{~m}, 1 \mathrm{H}, \mathrm{H}^{\mathrm{C} 3 \mathrm{~b}}\right), 5.85\left(\mathrm{~m}, 2 \mathrm{H}, \mathrm{H}^{\mathrm{D} 2 \mathrm{~b}}\right), 5.49$ (broad overlapping $\left.\mathrm{m}, 4 \mathrm{H}, \mathrm{H}^{\mathrm{D} 2 \mathrm{c}+\mathrm{D} 3 \mathrm{c}}\right), 2.03\left(\mathrm{~s}, 3 \mathrm{H}, \mathrm{H}^{\mathrm{Me}}\right), 1.28\left(\mathrm{~s}, 3 \mathrm{H}, \mathrm{H}^{\mathrm{Me}}\right) \cdot{ }^{13} \mathrm{C}$ NMR $\left(151 \mathrm{MHz}\right.$, acetone- $\left.d_{6}, 238 \mathrm{~K}\right) \delta / \mathrm{ppm}: 160.3\left(\mathrm{C}^{\mathrm{B} 6}\right), 154.9$ $\left(\mathrm{C}^{\mathrm{C} 1 \mathrm{~b}}\right), 154.5\left(\mathrm{C}^{\mathrm{Cla}}\right), 153.2\left(\mathrm{C}^{\mathrm{A} 2}\right), 152.9\left(\mathrm{C}^{\mathrm{B} 2}\right), 147.6\left(\mathrm{C}^{\mathrm{A} 6}\right)$, $139.7\left(\mathrm{C}^{\mathrm{B} 4}\right), 139.4\left(\mathrm{C}^{\mathrm{A} 4}\right), 135.9\left(\mathrm{C}^{\mathrm{P} 1}\right), 134.4\left(\mathrm{C}^{\mathrm{D} 4 \mathrm{c}}\right), 134.2$ $\left(\mathrm{C}^{\mathrm{C} 6 \mathrm{a}+\mathrm{C} 6 \mathrm{~b}}\right), 133.5\left(\mathrm{C}^{\mathrm{D} 2 \mathrm{~b}}\right), 133.3\left(\mathrm{C}^{\mathrm{D} 2 \mathrm{a}}\right), 132.3\left(\mathrm{C}^{\mathrm{P3a}}\right), 131.8$ $\left(\mathrm{C}^{\mathrm{P} 5 \mathrm{a}}\right), 131.7\left(\mathrm{C}^{\mathrm{D} 1 \mathrm{~d}}\right), 131.6\left(\mathrm{C}^{\mathrm{D} 1 \mathrm{a}}\right), 131.5\left(\mathrm{C}^{\mathrm{D} 2 \mathrm{c}+\mathrm{D} 3 \mathrm{c}}\right), 131.2$ $\left(\mathrm{C}^{\mathrm{D} 4 \mathrm{~d}}\right), 131.1\left(\mathrm{C}^{\mathrm{C} 3 \mathrm{~b}}\right), 131.0\left(\mathrm{C}^{\mathrm{P} 8 \mathrm{a}}\right), 130.8\left(\mathrm{C}^{\mathrm{C} 3 \mathrm{a}}\right), 130.6\left(\mathrm{C}^{\mathrm{D} 4 \mathrm{a}}\right)$, $129.8\left(\mathrm{C}^{\mathrm{D} 4 \mathrm{~b}}\right), 129.5\left(\mathrm{C}^{\mathrm{B} 5}\right), 129.46\left(\mathrm{C}^{\mathrm{D} 3 \mathrm{~d}}\right), 129.4\left(\mathrm{C}^{\mathrm{D} 3 \mathrm{a}}\right), 129.0$ $\left(\mathrm{C}^{\mathrm{P} 5+\mathrm{D} 2 \mathrm{~d}}\right), 128.9 \quad\left(\mathrm{C}^{\mathrm{P9}}\right), 128.5 \quad\left(\mathrm{C}^{\mathrm{P} 10 \mathrm{a}}\right), 128.3 \quad\left(\mathrm{C}^{\mathrm{D} 1 \mathrm{~b}}\right), 128.0$ $\left(\mathrm{C}^{\mathrm{D} 3 \mathrm{~b}+\mathrm{P} 4}\right), 127.7\left(\mathrm{C}^{\mathrm{C} 5 \mathrm{a}+\mathrm{C} 5 \mathrm{~b}}\right), 127.4\left(\mathrm{C}^{\mathrm{P} 7}\right), 127.1\left(\mathrm{C}^{\mathrm{P} 2}\right), 126.6$ $\left(\mathrm{C}^{\mathrm{P} 6}\right), 126.4\left(\mathrm{C}^{\mathrm{P} 8}\right), 126.0\left(\mathrm{C}^{\mathrm{P} 3}\right), 125.8\left(\mathrm{C}^{\mathrm{C} 4 \mathrm{~b}}\right), 125.7\left(\mathrm{C}^{\mathrm{A} 5}\right), 125.8$ $\left(\mathrm{C}^{\mathrm{C} 4 \mathrm{a}}\right), 124.8\left(\mathrm{C}^{\mathrm{P} 10 \mathrm{~b}}\right), 124.7\left(\mathrm{C}^{\mathrm{P} 10 \mathrm{c}}\right), 124.6\left(\mathrm{C}^{\mathrm{P} 10}\right), 124.3\left(\mathrm{C}^{\mathrm{A} 3}\right)$, $160.3\left(\mathrm{C}^{\mathrm{B} 6}\right), 123.1\left(\mathrm{C}^{\mathrm{B} 3}\right), 121.1\left(\mathrm{C}^{\mathrm{C} 2 \mathrm{a}}\right), 119.9\left(\mathrm{C}^{\mathrm{C} 2 \mathrm{~b}}\right), 36.5\left(\mathrm{C}^{\mathrm{q}}\right)$, $31.6\left(\mathrm{C}^{\mathrm{Me}}\right), 22.6\left(\mathrm{C}^{\mathrm{Me}}\right) .{ }^{31} \mathrm{P}$ NMR $\left(162 \mathrm{MHz}\right.$, acetone- $d_{6}, 295$ K) $\delta / \mathrm{ppm}-12.0($ broad, FWHM $=\sim 550 \mathrm{~Hz}),-144.2$ (septet, $J_{\mathrm{PF}}$ $\left.=707 \mathrm{~Hz},\left[\mathrm{PF}_{6}\right]\right) ;\left(243 \mathrm{MHz}\right.$, acetone- $\left.d_{6}, 238 \mathrm{~K}\right) \delta / \mathrm{ppm}-10.9$ (broad d, $J=95 \mathrm{~Hz}$ ), -13.5 (broad d, $J=95 \mathrm{~Hz}$ ). ESI MS: $m / z$ $997.3\left[\mathrm{M}-\mathrm{PF}_{6}\right]^{+}$(base peak, calc. 997.2). UV-Vis $\left(\mathrm{CH}_{2} \mathrm{Cl}_{2}, 2.5\right.$ $\left.\times 10^{-5} \mathrm{~mol} \mathrm{dm}{ }^{-3}\right): \lambda / \mathrm{nm}\left(\varepsilon / \mathrm{dm}^{3} \mathrm{~mol}^{-1} \mathrm{~cm}^{-1}\right) 243$ (68000), 280 (45000), 337sh (20000), 351 (24000). Found: C 67.51, H 4.81, $\mathrm{N} 2.59 ; \mathrm{C}_{65} \mathrm{H}_{48} \mathrm{CuF}_{6} \mathrm{~N}_{2} \mathrm{OP}_{3}$ requires $\mathrm{C} 68.27, \mathrm{H} 4.23, \mathrm{~N} 2.45 \%$. 
Table 1 Crystallographic data

\begin{tabular}{|c|c|c|c|c|c|}
\hline Compound & $\begin{array}{l}{[\mathrm{Cu}(1-\mathrm{Naphbpy})} \\
(\mathrm{POP})]\left[\mathrm{PF}_{6}\right] \cdot 0.25 \mathrm{THF}\end{array}$ & $\begin{array}{l}{[\mathrm{Cu}(2-\mathrm{Naphbpy})} \\
(\mathrm{POP})]\left[\mathrm{PF}_{6}\right]^{\cdot} \mathrm{Me}_{2} \mathrm{CO}\end{array}$ & 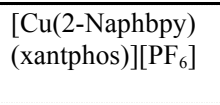 & $\begin{array}{l}{[\mathrm{Cu}(1-\mathrm{Pyrbpy})(\mathrm{POP})]} \\
{\left[\mathrm{PF}_{6}\right]^{\circ} 0.5 \mathrm{Et}_{2} \mathrm{O}^{\circ} \mathrm{CH}_{2} \mathrm{Cl}_{2}}\end{array}$ & $\begin{array}{l}{[\mathrm{Cu}(1-\mathrm{Pyrbpy})} \\
\left.\text { (xantphos)][} \mathrm{PF}_{6}\right] \\
0.5 \mathrm{Et}_{2} \mathrm{O}\end{array}$ \\
\hline Formula & $\mathrm{C}_{57} \mathrm{H}_{44} \mathrm{CuF}_{6} \mathrm{~N}_{2} \mathrm{O}_{1.25} \mathrm{P}_{3}$ & $\mathrm{C}_{59} \mathrm{H}_{48} \mathrm{CuF}_{6} \mathrm{~N}_{2} \mathrm{O}_{2} \mathrm{P}_{3}$ & $\mathrm{C}_{59} \mathrm{H}_{46} \mathrm{CuF}_{6} \mathrm{~N}_{2} \mathrm{OP}_{3}$ & $\mathrm{C}_{65} \mathrm{H}_{51} \mathrm{Cl}_{2} \mathrm{CuF}_{6} \mathrm{~N}_{2} \mathrm{O}_{1.5} \mathrm{P}_{3}$ & $\mathrm{C}_{67} \mathrm{H}_{53} \mathrm{CuF}_{6} \mathrm{~N}_{2} \mathrm{O}_{1.5} \mathrm{P}_{3}$ \\
\hline Formula weight & 1047.44 & 1087.50 & 1069.48 & 1225.49 & 1180.63 \\
\hline $\begin{array}{l}\text { Crystal colour and } \\
\text { habit }\end{array}$ & Yellow block & Yellow block & Yellow plate & Yellow block & Yellow block \\
\hline Crystal system & Orthorhombic & Monoclinic & Monoclinic & Monoclinic & Triclinic \\
\hline Space group & Pccn & $P 2_{1} / c$ & $P n$ & $C 2 / m$ & $P-1$ \\
\hline \multirow{3}{*}{$a, b, c / \AA$} & $22.6154(14)$ & $22.2781(11)$ & $11.4374(8)$ & $23.5648(17)$ & $13.0030(9)$ \\
\hline & $20.0116(12)$ & $14.7143(8)$ & $13.1660(9)$ & $43.959(3)$ & $14.4788(10)$ \\
\hline & $21.8373(14)$ & $15.4481(7)$ & $16.8565(12)$ & $12.3463(9)$ & $16.9143(11)$ \\
\hline \multirow[t]{3}{*}{$\alpha, \beta, \gamma /^{\circ}$} & 90 & 90 & 90 & 90 & $72.110(3)$ \\
\hline & 90 & $99.249(3)$ & $98.647(3)$ & $94.788(3)$ & $84.932(4)$ \\
\hline & 90 & 90 & 90 & 90 & $75.976(4)$ \\
\hline$U / \AA^{3}$ & $9882.9(11)$ & 4998.1(4) & $2509.5(3)$ & $12744.7(16)$ & $2939.8(4)$ \\
\hline$D_{c} / \mathrm{Mg} \mathrm{m}^{-3}$ & 1.408 & 1.445 & 1.415 & 1.277 & 1.334 \\
\hline$Z$ & 8 & 4 & 2 & 8 & 2 \\
\hline$\mu(\mathrm{Cu}-\mathrm{K} \alpha) / \mathrm{mm}^{-1}$ & 2.103 & 2.112 & 2.079 & 2.464 & 1.834 \\
\hline$T / \mathrm{K}$ & 123 & 123 & 123 & 123 & 123 \\
\hline Refln. collected $\left(R_{\text {int }}\right)$ & $70584(0.060)$ & $52879(0.055)$ & $16429(0.024)$ & $44681(0.032)$ & $39266(0.030)$ \\
\hline Unique refln. & 9287 & 9320 & 7635 & 11839 & 10819 \\
\hline Refln. for refinement & 7830 & 7470 & 7511 & 11247 & 9691 \\
\hline Parameters & 645 & 661 & 650 & 257 & 748 \\
\hline Threshold & $2 \sigma$ & $2 \sigma$ & $2 \sigma$ & $2 \sigma$ & $2 \sigma$ \\
\hline$R 1$ ( $R 1$ all data) & $0.0605(0.0713)$ & $0.0432(0.0572)$ & $0.0472(0.0476)$ & $0.0603(0.0622)$ & $0.0698(0.0761)$ \\
\hline$w R 2(w R 2$ all data) & $0.1485(0.1530)$ & $0.0866(0.1067)$ & $0.1120(0.1121)$ & $0.1527(0.1531)$ & $0.1719(0.1737$ \\
\hline Goodness of fit & 1.0589 & 0.8433 & 0.7142 & 1.0572 & 1.0983 \\
\hline CCDC deposition $t$ & 1528552 & 1528550 & 1528551 & 1528548 & 1528549 \\
\hline
\end{tabular}

Crystallography. Data were collected on a Bruker Kappa Apex2 diffractometer with data reduction, solution and refinement using the programs APEX $^{26}$ and CRYSTALS. ${ }^{27}$ Structural analysis was carried out using Mercury v. 3.7. ${ }^{28,29}$ Crystallographic data are summarized in Table 1.

\section{Results and discussion}

\section{Synthesis of $\mathrm{N}^{\wedge} \mathrm{N}$ ligands and of the copper(I) complexes}

The ligand 2-Naphbpy has previously been reported. ${ }^{25,30}$ However, we found that the palladium-catalysed coupling of 6bromo-2,2'-bipyridine with 2-naphthaleneboronic acid (using the second generation Suzuki-Miyaura precatalyst SPhosPd G2) was a more convenient synthetic strategy than the literature methods. An analogous procedure was used to prepare 2Naphbpy and 1-Pyrbpy. Each ligand was characterized by ${ }^{1} \mathrm{H}$ and ${ }^{13} \mathrm{C}$ NMR spectroscopies and mass spectrometry (see Experimental section).

Two different strategies are typically used to prepare the heteroleptic complexes $\left[\mathrm{Cu}\left(\mathrm{N}^{\wedge} \mathrm{N}\right)\left(\mathrm{P}^{\wedge} \mathrm{P}\right)\right]\left[\mathrm{PF}_{6}\right]\left(\mathrm{P}^{\wedge} \mathrm{P}=\mathrm{POP}\right.$ or xantphos). The addition of $\mathrm{POP}$ to a solution of $\left[\mathrm{Cu}(\mathrm{MeCN})_{4}\right]\left[\mathrm{PF}_{6}\right]$ leads to the formation of three-coordinate complexes $[\mathrm{Cu}(\mathrm{POP})(\mathrm{MeCN})]^{+}$or $\left[\mathrm{Cu}\left(\mathrm{POP}-P_{,} P^{\prime}\right)(\mathrm{POP}-P)\right]^{+}$. The steric demands of $\mathrm{POP}$ prevent the formation of the fourcoordinate $\left[\mathrm{Cu}\left(\mathrm{POP}-P, P^{\prime}\right)_{2}\right]^{+}{ }^{31}$ The monodentate ligand in the three-coordinate intermediate is readily substituted by an $\mathrm{N}^{\wedge} \mathrm{N}$ ligand such as bpy. ${ }^{6}$ With the less sterically demanding ligand xantphos, the formation of a homoleptic bis-chelate complex $\left[\mathrm{Cu}\left(\text { xantphos }-P, P^{\prime}\right)_{2}\right]^{+}$is more likely. The optimized strategy for $\left[\mathrm{Cu}\left(\mathrm{N}^{\wedge} \mathrm{N}\right)(\mathrm{POP})\right]\left[\mathrm{PF}_{6}\right]$ complexes is sequential addition of

1-1.2 equivalents of POP followed after $\approx 2$ hours by a bpy ligand to a solution of $\left[\mathrm{Cu}(\mathrm{MeCN})_{4}\right]\left[\mathrm{PF}_{6}\right]$. For $\left[\mathrm{Cu}\left(\mathrm{N}^{\wedge} \mathrm{N}\right)(\right.$ xantphos) $]\left[\mathrm{PF}_{6}\right]$, a $1: 1$ solution of $\mathrm{N}^{\wedge} \mathrm{N}$ and xantphos is added to a solution of $\left[\mathrm{Cu}(\mathrm{MeCN})_{4}\right]\left[\mathrm{PF}_{6}\right]$. Immediately after mixing, the solution may appear red, indicating the presence of some $\left[\mathrm{Cu}\left(\mathrm{N}^{\wedge} \mathrm{N}\right)_{2}\right]^{+}$complex. However, after stirring for 2 hours, equilibration yields yellow $\left[\mathrm{Cu}\left(\mathrm{N}^{\wedge} \mathrm{N}\right)(\text { xantphos })\right]^{+}$as the preferred solution species.

The complexes $[\mathrm{Cu}(1-\mathrm{Naphbpy})(\mathrm{POP})]\left[\mathrm{PF}_{6}\right], \quad[\mathrm{Cu}(2-$ Naphbpy)(POP) $]\left[\mathrm{PF}_{6}\right], \quad[\mathrm{Cu}(1-\mathrm{Pyrbpy})(\mathrm{POP})]\left[\mathrm{PF}_{6}\right], \quad[\mathrm{Cu}(1-$

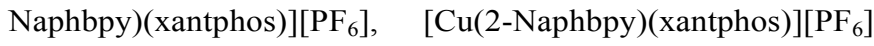
and $[\mathrm{Cu}(1-\mathrm{Pyrbpy})(\mathrm{xantphos})]\left[\mathrm{PF}_{6}\right]$ were isolated as yellow solids in yields of between 38 and $98 \%$. The electrospray mass spectrum of each complex showed a peak corresponding to [M$\left.\mathrm{PF}_{6}\right]^{+}$with a characteristic isotope pattern. The presence of the $\left[\mathrm{PF}_{6}\right]^{-}$counterion was confirmed by the appearance of a septet at $\delta-144.4 \mathrm{ppm}$ in the ${ }^{31} \mathrm{P}$ NMR spectrum, in addition to the signals for the POP or xantphos ligands (see later). 


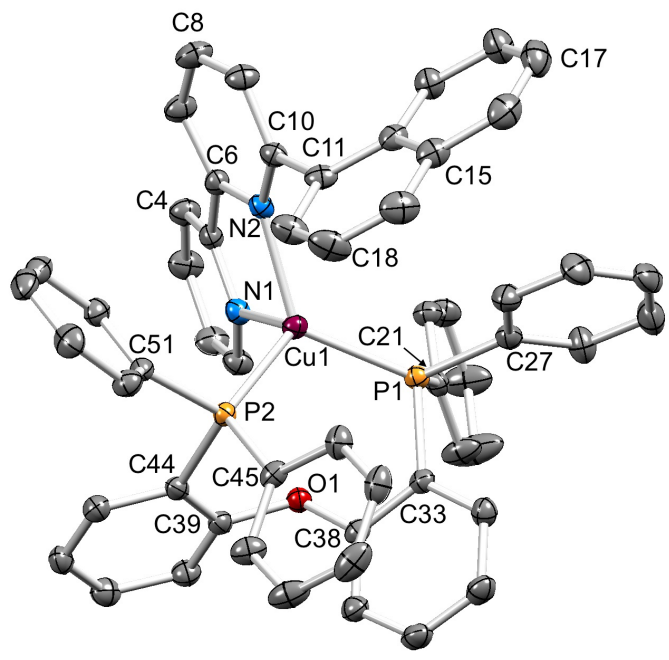

Fig. 2. Structure of the $[\mathrm{Cu}(1-\mathrm{Naphbpy})(\mathrm{POP})]^{+}$cation in $[\mathrm{Cu}(1-$ Naphbpy)(POP)][PF $]_{6} \cdot 0.25 \mathrm{THF}$ with ellipsoids plotted at $40 \%$ probability level. $\mathrm{H}$ atoms and solvent molecules are omitted for clarity. Selected bond parameters: Cu1-P1 = 2.2723(8), Cu1-P2 = 2.2833(8), Cu1-N1 = 2.118(2), Cu1-N2 = 2.111(2) Å; P1-Cu1-P2 = 109.97(3), P1-Cu1-N1 = 106.14(7), P2-Cu1-N1 = 109.85(7), P1$\mathrm{Cu} 1-\mathrm{N} 2=128.14(7), \mathrm{P} 2-\mathrm{Cu} 1-\mathrm{N} 2=116.14(7), \mathrm{N} 1-\mathrm{Cu} 1-\mathrm{N} 2=79.68(9)^{\circ}$.

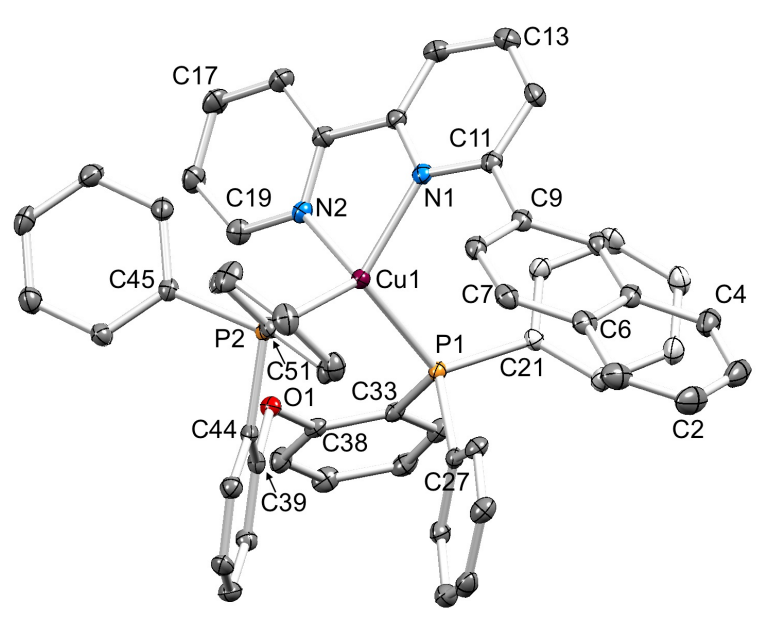

Fig. 3. Structure of the $[\mathrm{Cu}(2-\mathrm{Naphbpy})(\mathrm{POP})]^{+}$cation in $[\mathrm{Cu}(2-$ Naphbpy)(POP)] $\left[\mathrm{PF}_{6}\right]^{\cdot} \mathrm{Me}_{2} \mathrm{CO}$ with ellipsoids plotted at $40 \%$ probability level. $\mathrm{H}$ atoms and solvent molecules are omitted for clarity. Selected bond parameters: Cu1-P1 = 2.2600(8), Cu1-P2 = 2.2716(8), Cu1-N1 = 2.105(2), Cu1-N2 = 2.099(2) Å; P1-Cu1-P2 = 112.01(3), P1-Cu1-N1 = 109.59(6), P2-Cu1-N1 = 131.94(6), P1Cu1-N2 $=113.42(7)$, P2-Cu1-N2 $=104.26(7), N 1-C u 1-N 2=79.68(9)^{\circ}$.

\section{Single crystal structures}

Single crystals of $[\mathrm{Cu}(1-\mathrm{Naphbpy})(\mathrm{POP})]\left[\mathrm{PF}_{6}\right]^{\circ} 0.25 \mathrm{THF}$, [Cu(2-Naphbpy)(POP) $]\left[\mathrm{PF}_{6}\right]^{\cdot} \mathrm{Me}_{2} \mathrm{CO}$,

$[\mathrm{Cu}(1-$ Pyrbpy)(POP) $]\left[\mathrm{PF}_{6}\right]^{0} 0.5 \mathrm{Et}_{2} \mathrm{O} \cdot \mathrm{CH}_{2} \mathrm{Cl}_{2}$, $[\mathrm{Cu}(2-$ Naphbpy)(xantphos) $]\left[\mathrm{PF}_{6}\right]$ and $[\mathrm{Cu}(1-$ Pyrbpy)(xantphos) $]\left[\mathrm{PF}_{6}\right]^{\circ} 0.5 \mathrm{Et}_{2} \mathrm{O}$ were grown by diffusion of $\mathrm{Et}_{2} \mathrm{O}$ into solutions of the complexes in THF ([Cu(1Naphbpy)(POP) $\left.]\left[\mathrm{PF}_{6}\right]\right), \mathrm{Me}_{2} \mathrm{CO}$ ([Cu(2-Naphbpy)(POP)][PF $]$ ) or $\mathrm{CH}_{2} \mathrm{Cl}_{2}$ (the remaining complexes). The structures exhibit many similarities and it is appropriate to discuss them in a comparative manner. Figs. 2-6 show the structures of the $[\mathrm{Cu}(1-\mathrm{Naphbpy})(\mathrm{POP})]^{+}, \quad[\mathrm{Cu}(2-\mathrm{Naphbpy})(\mathrm{POP})]^{+}, \quad[\mathrm{Cu}(2-$ Naphbpy)(xantphos) $]^{+}, \quad[\mathrm{Cu}(1-\mathrm{Pyrbpy})(\mathrm{POP})]^{+}$and $[\mathrm{Cu}(1-$ Pyrbpy)(xantphos) $]^{+}$cations, respectively, and parameters describing the copper(I) coordination environments are given in the figure captions. In each complex, the copper(I) atom is in a distorted tetrahedral environment with $\mathrm{Cu}-\mathrm{P}$ and $\mathrm{Cu}-\mathrm{N}$ bond lengths in the ranges 2.2453(10)-2.3101(11) and 2.085(4)2.143(3) $\AA$, respectively. The $\mathrm{P}-\mathrm{Cu}-\mathrm{P}$ angles fall in the range 109.97(3)-116.83(4) $)^{\circ}$, the largest being for the $[\mathrm{Cu}(2-$ Naphbpy)(xantphos) $]^{+}$cation. As expected, the $\mathrm{N}-\mathrm{Cu}-\mathrm{N}$ bond angles vary little across the series $\left(79.16(14)-79.88(13)^{\circ}\right)$. The asymmetry of the 6-substituted bpy ligand means that there are two possible orientations of the $\mathrm{N}^{\wedge} \mathrm{N}$ ligand with respect to the $\mathrm{P}^{\wedge} \mathrm{P}$ ligand, one with the 6-substituent lying over the 'xanthene bowl' and one with it positioned remote from the bowl as shown in Fig. 1. The two orientations are related by a $180^{\circ}$ rotation of the bpy unit. We have previously reported the solidstate structures of $[\mathrm{Cu}(6-\mathrm{Mebpy})(\mathrm{xantphos})]^{+}$and $[\mathrm{Cu}(6-$ Etbpy)(xantphos) $]^{+}$in which the 6-alkyl substituent lies over the 'bowl' of the xanthene unit (Fig. 1b); in contrast, the 6phenyl substituent in $[\mathrm{Cu}(6-\mathrm{Phbpy})(\mathrm{xantphos})]^{+}$is remote from the xanthene 'bowl' (Fig. 1a). ${ }^{8}$ Each of the cations shown in Fig. 2-6 exhibits the same $\mathrm{N}^{\wedge} \mathrm{N}$ ligand orientation with the sterically-demanding naphthyl or pyrenyl group positioned away from the $\left(\mathrm{C}_{6} \mathrm{H}_{4}\right)_{2} \mathrm{O}$ unit of POP or the xanthene 'bowl' of xantphos (Fig. 7a). We return to this point in the NMR spectroscopic discussion.

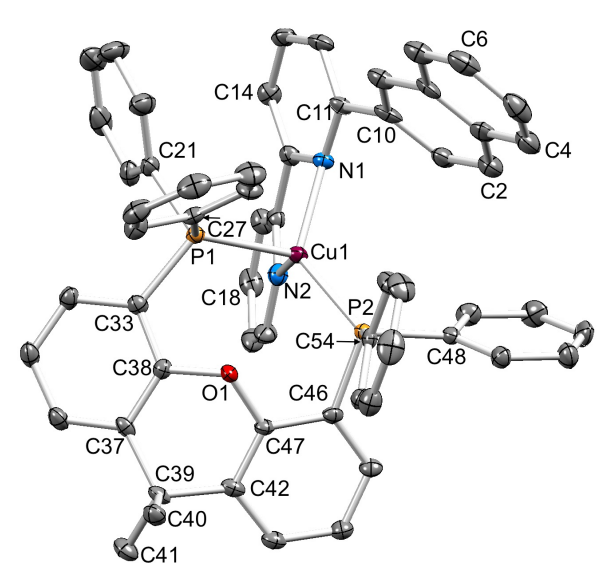

Fig. 4. Structure of the $[\mathrm{Cu}(2-\mathrm{Naphbpy})(\text { xantphos })]^{+}$cation in $[\mathrm{Cu}(2-$ Naphbpy)(xantphos)][ $\left.\mathrm{PF}_{6}\right]$ with ellipsoids plotted at $30 \%$ probability level. $\mathrm{H}$ atoms omitted for clarity. Selected bond parameters: Cu1-P2 $=2.2453(10)$, Cu1$\mathrm{P} 1=2.3101(11), \mathrm{Cu} 1-\mathrm{N} 2=2.085(4), \mathrm{Cu} 1-\mathrm{N} 1=2.143(3) \AA \AA$ 116.83(4), P2-Cu1-N2 = 113.47(10), P1-Cu1-N2 = 105.84(10), P2-Cu1-N1 = 130.15(10), P1-Cu1-N1 = 103.74(10), N2-Cu1-N1 = 79.16(14) ${ }^{\circ}$. 


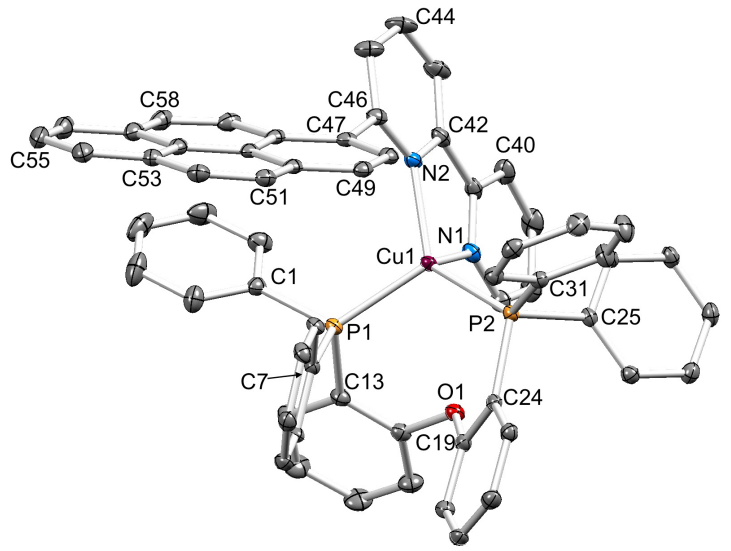

Fig. 5. Structure of the $[\mathrm{Cu}(1-\mathrm{Pyrbpy})(\mathrm{POP})]^{+}$cation in $[\mathrm{Cu}(1-$ Pyrbpy)(POP)] $\left[\mathrm{PF}_{6}\right] 0.5 \mathrm{Et}_{2} \mathrm{O} \mathrm{CH}_{2} \mathrm{Cl}_{2}$ with ellipsoids plotted at $30 \%$ probability level. $\mathrm{H}$ atoms and solvent molecules omitted for clarity. Selected bond parameters: $\mathrm{Cu} 1-\mathrm{P} 1=2.2543(7), \mathrm{Cu} 1-\mathrm{P} 2=2.2876(7), \mathrm{Cu} 1-\mathrm{N} 1=2.098(2), \mathrm{Cu} 1-\mathrm{N} 2=2.089(2)$ Å; P1-Cu1-P2 = 112.04(2), P1-Cu1-N1 = 111.02(6), P2-Cu1-N1 = 104.99(6), P1$\mathrm{Cu} 1-\mathrm{N} 2=114.25(6), \mathrm{P} 2-\mathrm{Cu} 1-\mathrm{N} 2=127.85(6), \mathrm{N} 1-\mathrm{Cu} 1-\mathrm{N} 2=79.52(8)^{\circ}$.

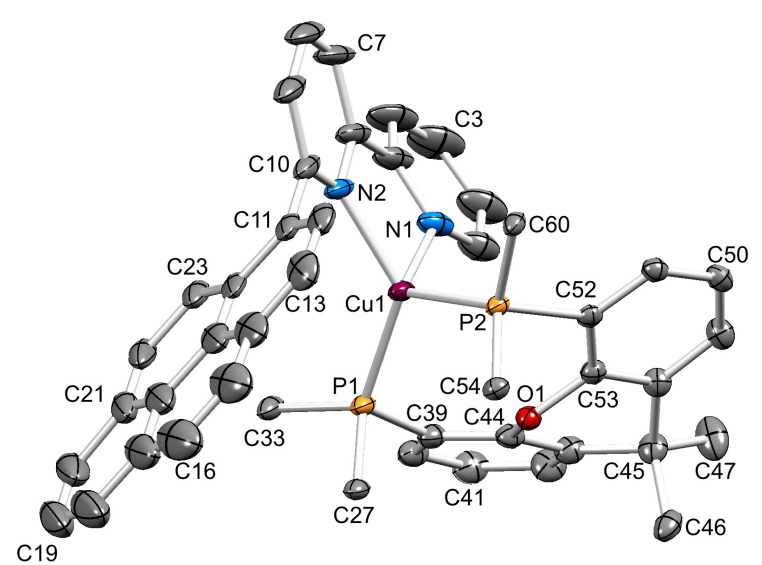

Fig. 6. Structure of the $[\mathrm{Cu}(1-\mathrm{Pyrbpy})(\mathrm{xantphos})]^{+}$cation in $[\mathrm{Cu}(1$ Pyrbpy)(xantphos)][ $\left[\mathrm{PF}_{6}\right] \cdot 0.5 \mathrm{Et}_{2} \mathrm{O}$ with ellipsoids plotted at $30 \%$ probability level. $\mathrm{H}$ atoms and solvent molecules omitted for clarity, and only the ipso-C atom of each of the four P-attached phenyl rings is shown. Selected bond parameters: $\mathrm{Cu} 1-\mathrm{P} 2=2.2576(7), \mathrm{Cu} 1-\mathrm{P} 1=2.2649(8), \mathrm{Cu} 1-\mathrm{N} 2=2.098(2), \mathrm{Cu} 1-\mathrm{N} 1=2.104(3)$ Å; P2-Cu1-P1 = 112.20(3), P2-Cu1-N2 = 110.70(8), P1-Cu-N2 = 130.64(7), P2Cu1-N1 = 112.49(8), P1-Cu1-N1 = 104.67(8), N2-Cu1-N1 = 79.88(13) ${ }^{\circ}$.

The interannular twist within the bpy unit varies significantly across the series of structures with the angle between the planes through the pyridine rings ranging from 5.8 to $26.0^{\circ}$ (Table 2). This twist is coupled with the orientation of the pendant aryl unit with respect to the phenyl substituents of the POP or xantphos ligand. In [Cu(1-Pyrbpy)(xantphos) $]^{+}$and $[\mathrm{Cu}(2-\mathrm{Naphbpy}) \text { (xantphos) }]^{+}$, one phenyl ring of each $\mathrm{PPh}_{2}$ group engages in an edge-to-face interaction with the pyrenyl or naphthyl group (Fig. 7). The closest $\mathrm{CH} \ldots \pi$ contacts $^{32}$ (measured to the centroid of the closest $\mathrm{C}_{6}$ ring) are $2.75 \AA$ in $[\mathrm{Cu}(2-\mathrm{Naphbpy}) \text { (xantphos) }]^{+}$and 2.45 and $2.58 \AA$ in $[\mathrm{Cu}(1-$ Pyrbpy)(xantphos) $]^{+}$. Each POP-containing complex exhibits face-to-face $\pi$-stacking between one arene ring of the $\left(\mathrm{C}_{6} \mathrm{H}_{4}\right)_{2} \mathrm{O}$ unit and one phenyl ring (Fig. 8). In [Cu(1-Naphbpy)(POP) $]^{+}$ (Fig. 8a), this interaction is characterized by an angle between ring planes, centroid...centroid distance, and centroid...plane separation of $12.0^{\circ}, 3.56 \AA$ and $3.53 \AA$. The angles between the ring planes in $[\mathrm{Cu}(1-\mathrm{Pyrbpy})(\mathrm{POP})]^{+}$and $[\mathrm{Cu}(2-$ Naphbpy)(POP) $]^{+}$are, however, 26.0 and $23.4^{\circ}$, respectively. This deviation from a coplanar orientation is associated with edge-to-face $\mathrm{CH} . . . \pi$ contacts involving the 6-substituted pyrenyl or naphthyl group (Fig. 8b and 8c).

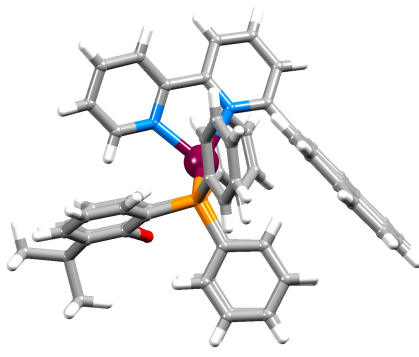

(a)

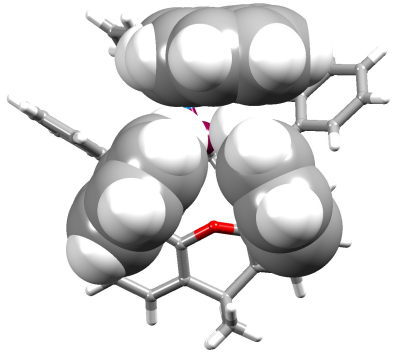

(b)
Fig. 7. (a) The $[\mathrm{Cu}(1-\mathrm{Pyrbpy})(x a n t p h o s)]^{+}$cation showing orientation of the 1pyrenyl substituent (right) remote from the xanthene 'bowl' (left). (b) Edge-toface positioning of the two phenyl rings of xantphos and the naphthyl unit in [Cu(2-Naphbpy)(xantphos) $]^{+}$.

Interestingly, intermolecular face-to-face $\pi$-contacts play an insignificant role in packing in the solid state. Pyrenyl-units of

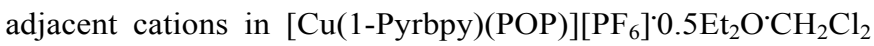
approach closely (Fig. 9), but the orientation of the two arene moieties is not optimal for efficient stacking. The angle between the least squares planes through the two pyrenyl units is $21.8^{\circ}$ and the centroid...centroid separation of the two closest $\mathrm{C}_{6}$-rings is $3.80 \AA$.

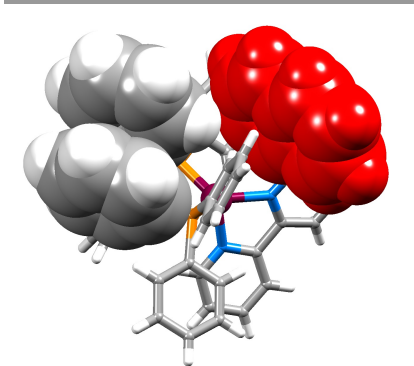

(a)

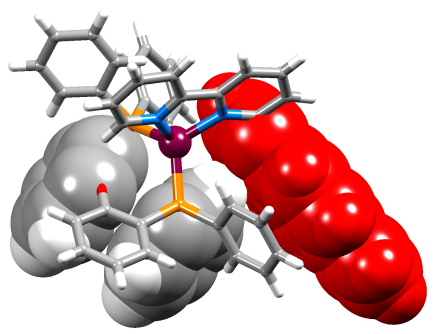

(b)

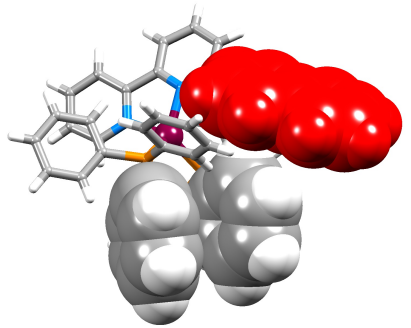

(c)

Fig. 8. Structures of (a) $[\mathrm{Cu}(1-\mathrm{Naphbpy})(\mathrm{POP})]^{+}$, (b) $[\mathrm{Cu}(1-\mathrm{Pyrbpy})(\mathrm{POP})]^{+}$and (c) $[\mathrm{Cu}(2-\mathrm{Naphbpy})(\mathrm{POP})]^{+}$showing $\pi$-stacking of arene rings within the POP ligand. See text discussion. The 6-substituted naphthyl or pyrenyl group is shown in red. 
Table 2. Angular distortions of the $\mathrm{N}^{\wedge} \mathrm{N}$ ligands in the $\left[\mathrm{Cu}\left(\mathrm{N}^{\wedge} \mathrm{N}\right)\left(\mathrm{P}^{\wedge} \mathrm{P}\right)\right]^{+}$ cations.

\begin{tabular}{|c|c|c|}
\hline Complex & $\begin{array}{c}\text { Angle between } \\
\text { planes of } \\
\text { pyridine rings } /{ }^{\circ}\end{array}$ & $\begin{array}{c}\text { Angle between plane } \\
\text { of pyridine ring and } \\
\text { attached arene unit } /{ }^{\circ}\end{array}$ \\
\hline$\left[\mathrm{Cu}(1-\text { Naphbpy)(POP) }]^{+}\right.$ & 16.0 & 69.0 \\
\hline$\left[\mathrm{Cu}(2-\text { Naphbpy)(POP) }]^{+}\right.$ & 18.4 & 50.5 \\
\hline$\left[\mathrm{Cu}(1-\text { Pyrbpy)(POP) }]^{+}\right.$ & 5.8 & 66.6 \\
\hline$\left[\mathrm{Cu}(2-\text { Naphbpy)(xantphos) }]^{+}\right.$ & 22.8 & 44.0 \\
\hline$\left[\mathrm{Cu}(1-\text { Pyrbpy)(xantphos) }]^{+}\right.$ & 26.0 & 60.8 \\
\hline
\end{tabular}

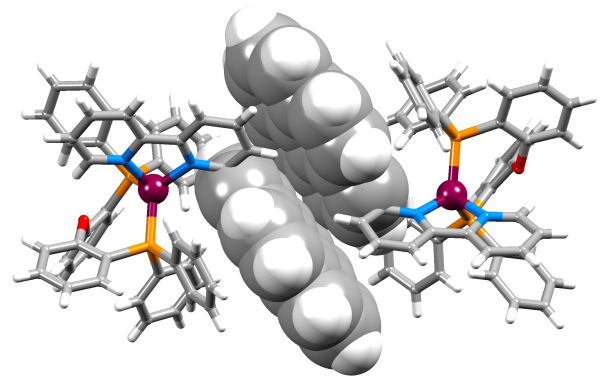

Fig. 9. Close approach of adjacent pyrenyl-units of adjacent [Cu(1-Pyrbpy)(POP)] cations (symmetry codes $x, y, z$ and $1-x, y, 1-z$ ).

\section{NMR spectroscopy and dynamic behaviour of the $\left[\mathrm{Cu}\left(\mathbf{N}^{\wedge} \mathbf{N}\right)\left(\mathbf{P}^{\wedge} \mathbf{P}\right)\right]\left[\mathrm{PF}_{6}\right]$ complexes}

Acetone- $d_{6}$ was chosen as the solvent for NMR spectroscopic studies because previous studies revealed that some $\left[\mathrm{Cu}\left(\mathrm{N}^{\wedge} \mathrm{N}\right)\left(\mathrm{P}^{\wedge} \mathrm{P}\right)\right]^{+}$complexes are prone to ligand dissociation in $\mathrm{CD}_{2} \mathrm{Cl}_{2}$ after several days in solution. ${ }^{8,11}$ Fig. 10 shows the room temperature ${ }^{1} \mathrm{H}$ NMR spectra of the six complexes. Although the resonances arising from the bpy domain are sharp in all cases, those from the 6-aryl substituent are only sharp at $298 \mathrm{~K}$ for the 2-Naphthyl group. Signals from the POP and xantphos ligands are broadened in $[\mathrm{Cu}(2-\mathrm{Naphbpy})(\mathrm{POP})]\left[\mathrm{PF}_{6}\right]$ and $[\mathrm{Cu}(2-\mathrm{Naphbpy})(\mathrm{xantphos})]\left[\mathrm{PF}_{6}\right]$, and are very broad for the remaining complexes (Fig. 10). NMR spectroscopic characterization of $[\mathrm{Cu}(2-\mathrm{Naphbpy})(\mathrm{POP})]\left[\mathrm{PF}_{6}\right]$ and $[\mathrm{Cu}(2-$ Naphbpy)(xantphos)][ $\left.\mathrm{PF}_{6}\right]$ was carried out by recording COSY, NOESY, HMBC and HMQC spectra at $298 \mathrm{~K}$. For the remaining complexes, ROESY, TOCSY, HSQC, HMBC and ${ }^{1} \mathrm{H}-{ }^{31} \mathrm{P}-\mathrm{HSQC}$ (only for the complexes containing 1-Pyrbpy) spectra were measured at lower temperatures. Use of these $2 \mathrm{D}$ methods permitted full assignment of the ${ }^{1} \mathrm{H}$ and ${ }^{13} \mathrm{C}$ NMR spectra (see Experimental section) and atom labelling is given in Sche

In the discussion below, we assume that dynamic behaviour arises from non-dissociative processes ${ }^{8}$ and initially consider the three complexes containing POP. In $[\mathrm{Cu}(2-$ Naphbpy)(POP)] $\left[\mathrm{PF}_{6}\right]$, one set of signals arising from the POPbackbone ring-C protons (correlating in the HMQC spectrum to one set of ${ }^{13} \mathrm{C}$ NMR signals) is observed at $298 \mathrm{~K}$. This is consistent with the $[\mathrm{Cu}(2-\mathrm{Naphbpy})(\mathrm{POP})]^{+}$cation having $C_{2}$ symmetry. Each $\mathrm{PPh}_{2}$ group of the POP ligand gives two sets of signals, one each for the phenyl rings (labelled $\mathrm{Da}$ and $\mathrm{Db}$ ) pointing towards or away from the aryl group on the 6-position of the bpy. The conformational flexibility of the 8-membered chelate $\{\mathrm{CuPCCOCCP}\}$-ring can exchange these positions as confirmed by exchange peaks (EXSY) in the ROESY spectrum between pairs $\mathrm{H}^{\mathrm{D} 2 \mathrm{a} /} \mathrm{H}^{\mathrm{D} 2 \mathrm{~b}}, \mathrm{H}^{\mathrm{D} 3 \mathrm{a}} \mathrm{H}^{\mathrm{D} 3 \mathrm{~b}} \mathrm{H}^{\mathrm{D} 4 \mathrm{a} /} \mathrm{H}^{\mathrm{D} 4 \mathrm{~b}}$ (Fig. 11). Low temperature ${ }^{13} \mathrm{C}$ and ${ }^{1} \mathrm{H} \quad \mathrm{NMR}$ spectra of $[\mathrm{Cu}(1-$ Naphbpy)(POP) $]\left[\mathrm{PF}_{6}\right]$ and $[\mathrm{Cu}(1-\mathrm{Pyrbpy})(\mathrm{POP})]\left[\mathrm{PF}_{6}\right]$ indicated loss of $C_{2}$ symmetry and as an example, we focus on $[\mathrm{Cu}(1-$ Naphbpy)(POP) $]\left[\mathrm{PF}_{6}\right]$. Two sets of signals for the $\mathrm{C}$ rings of the POP-backbone are observed (labelled $\mathrm{Ca}$ and $\mathrm{Cb}$, see Experimental Section) along with four sets of signals for the Pbonded phenyl rings (labelled $\mathrm{Da}, \mathrm{Db}, \mathrm{Dc}$ and $\mathrm{Dd}$ ). The ROESY spectrum (at $253 \mathrm{~K}$ ) is shown in Fig. S1†, and from this, EXSY cross-peaks the exchange of rings $\mathrm{Ca} / \mathrm{Cb}, \mathrm{Da} / \mathrm{Db}$ and Dc/Dd at higher temperatures.

mes 1 and 2 .

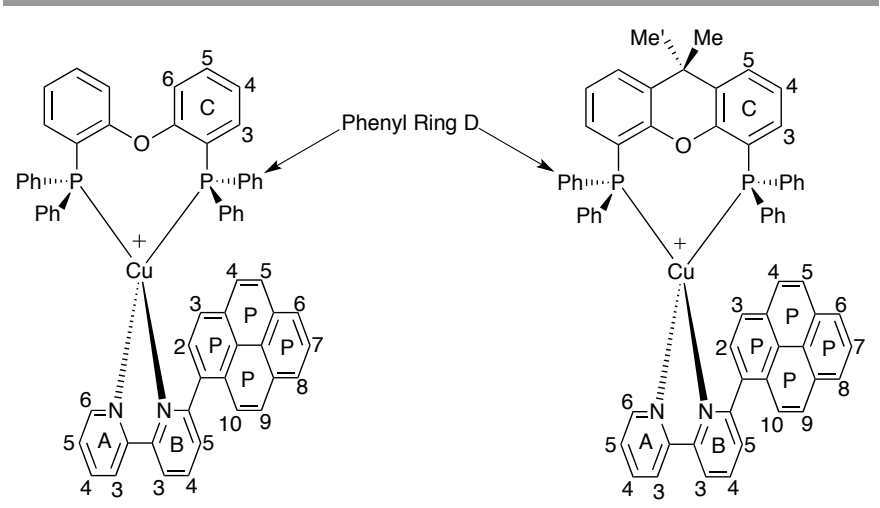

Scheme 2. Structures of $[\mathrm{Cu}(1-\mathrm{Pyr})(\mathrm{POP})]^{+}$(left) and $[\mathrm{Cu}(1-\mathrm{Pyr})(\mathrm{xantphos})]^{+}$with ring and atom labelling for NMR spectroscopic assignments.
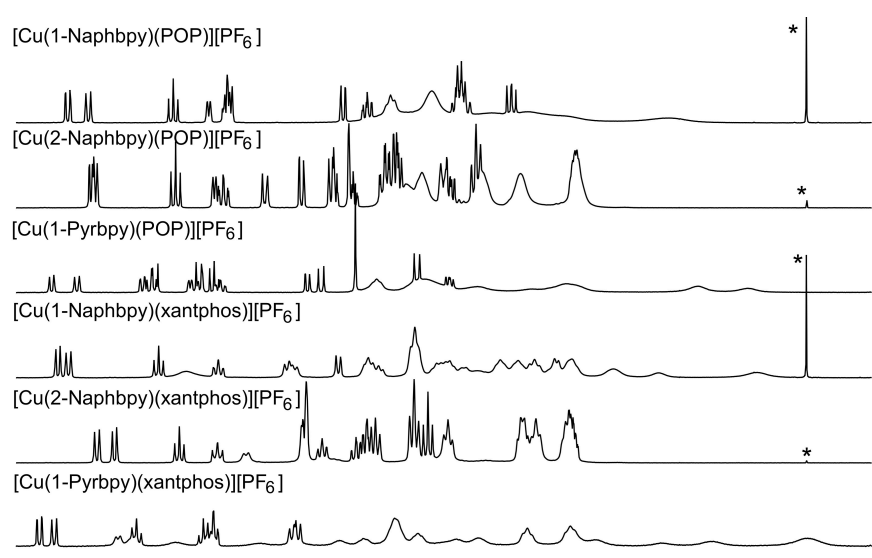

$\begin{array}{llllllllllllllllllll}8.8 & 8.6 & 8.4 & 8.2 & 8.0 & 7.8 & 7.6 & 7.4 & 7.2 & 7.0 & 6.8 & 6.6 & 6.4 & 6.2 & 6.0 & 5.8 & 5.6 & 5.4\end{array}$ Fig. 10. Room temperature $400 \mathrm{MHz}{ }^{1} \mathrm{H}$ NMR spectra of acetone- $d_{6}$ solutions of the six complexes. The alkyl regions of the spectra are omitted. ${ }^{*}=$ residual $\mathrm{CH}_{2} \mathrm{Cl}_{2}$. Chemical shifts in $\delta / \mathrm{ppm}$. 


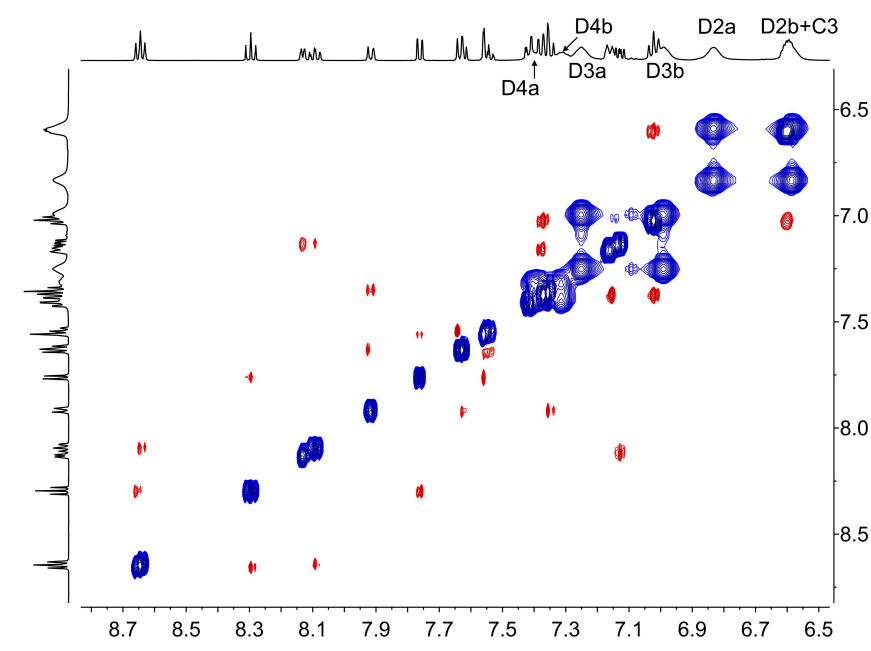

Fig. 11. $400 \mathrm{MHz}$ ROESY spectrum of [Cu(2-Naphbpy)(POP)] $\left[\mathrm{PF}_{6}\right]$ at $298 \mathrm{~K}$ in acetone- $d_{6}$. Signals in phase with the diagonal (blue) correspond to an EXSY peak and signals with opposite phase relative to the diagonal (red) correspond to and NOESY peak. Chemical shifts in $\delta / p p m$.

We now turn to the solution dynamic behaviour of $[\mathrm{Cu}(1-$ Naphbpy)(xantphos) $]^{+}, \quad[\mathrm{Cu}(2-\mathrm{Naphbpy})(\mathrm{xantphos})]^{+}$and $[\mathrm{Cu}(1-\mathrm{Pyrbpy})(\mathrm{xantphos})]^{+}$and Fig. 10 shows the room temperature ${ }^{1} \mathrm{H}$ NMR spectra. As observed for the POP complexes, the spectra for $[\mathrm{Cu}(1-\mathrm{Naphbpy})(\mathrm{xantphos})]\left[\mathrm{PF}_{6}\right]$ and $[\mathrm{Cu}(1-\mathrm{Pyrbpy})(\mathrm{xantphos})]\left[\mathrm{PF}_{6}\right]$ are very broad at $298 \mathrm{~K}$, indicating dynamic behaviour. Fig. 12 shows the effect of cooling an acetone- $d_{6}$ solution of $[\mathrm{Cu}(1-$ Pyrbpy)(xantphos)][ $\left.\mathrm{PF}_{6}\right]$; similar changes are observed for $[\mathrm{Cu}(1-\mathrm{Naphbpy})(\mathrm{xantphos})]\left[\mathrm{PF}_{6}\right]$. The spectra at $238 \mathrm{~K}\left(\mathrm{~N}^{\wedge} \mathrm{N}=\right.$ 1-Pyrbpy) or $223 \mathrm{~K}\left(\mathrm{~N}^{\wedge} \mathrm{N}=1\right.$-Naphbpy) were fully assigned using 2D methods (see Experimental Section).

$298 \mathrm{~K}$

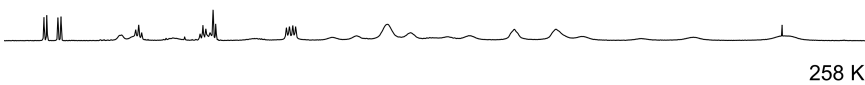

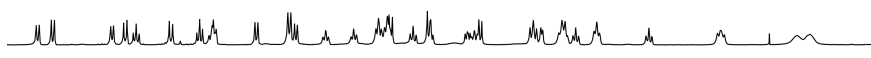

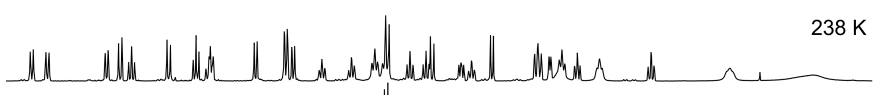

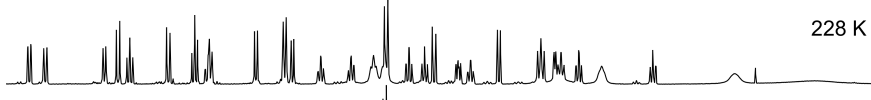

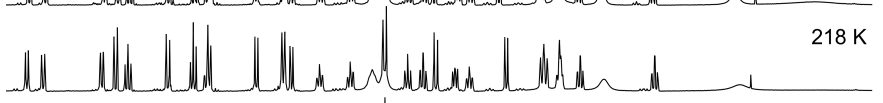

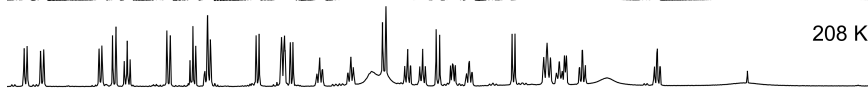

$\begin{array}{llllllllllllllllllll}8.9 & 8.7 & 8.5 & 8.3 & 8.1 & 7.9 & 7.7 & 7.5 & 7.3 & 7.1 & 6.9 & 6.7 & 6.5 & 6.3 & 6.1 & 5.9 & 5.7 & 5.5 & 5.3\end{array}$ Fig. 12. Variable temperature $600 \mathrm{MHz}^{1} \mathrm{H}$ NMR spectra of an acetone- $\mathrm{d}_{6}$ solution of $[\mathrm{Cu}(1-\mathrm{Pyr})(\mathrm{xantphos})]\left[\mathrm{PF}_{6}\right]$. The alkyl region of the spectrum is omitted. Chemical shifts in $\delta / p p m$.

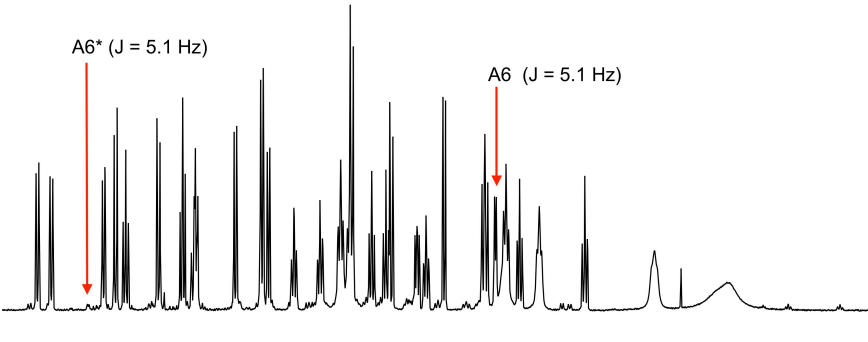

$\begin{array}{llllllllllllllllllllll}9.0 & 8.8 & 8.6 & 8.4 & 8.2 & 8.0 & 7.8 & 7.6 & 7.4 & 7.2 & 7.0 & 6.8 & 6.6 & 6.4 & 6.2 & 6.0 & 5.8 & 5.6 & 5.4 & 5.2 & 5.0\end{array}$ Fig. 13. Part of the solution $600 \mathrm{MHz}{ }^{1} \mathrm{H}$ NMR spectrum of $[\mathrm{Cu}(1-$ Pyr)(xantphos)][PF $\left[\right.$ (acetone- $\mathrm{d}_{6}$ ) at $238 \mathrm{~K}$. Chemical shifts in $\delta / \mathrm{ppm}$. See text for explanation of the subspectrum.

Although xantphos is more rigid than POP, the xanthene 'bowl' may undergo inversion. ${ }^{33}$ We previously reported that in solution, $[\mathrm{Cu} \text { (Phbpy)(xantphos) }]^{+}$(Phbpy $=6$-phenyl-2,2'bipyridine) exists as two conformers which are related by the inversion of the xanthene 'bowl' (Fig. 1c). The relative population of the conformers of $[\mathrm{Cu}(\mathrm{Phbpy})(\mathrm{xantphos})]^{+}$in $\mathrm{CD}_{2} \mathrm{Cl}_{2}$ was $\sim 1.0: 0.9$, and the calculated energy difference was $3.57 \mathrm{kcal} \mathrm{mol}{ }^{-1}$. The characteristic ${ }^{1} \mathrm{H}$ NMR signal distinguishing the two conformers is that for $\mathrm{H}^{\mathrm{A} 6}$ (see Scheme 2 and Fig. 1c for labelling) which appeared at $\delta 8.42 \mathrm{ppm}$ in one conformer and $\delta 6.35 \mathrm{ppm}$ in the other. ${ }^{8}$ In the low temperature ${ }^{1} \mathrm{H} \quad \mathrm{NMR}$ spectra of $[\mathrm{Cu}(1-\mathrm{Naphbpy})(\mathrm{xantphos})]\left[\mathrm{PF}_{6}\right]$ and $\left[\mathrm{Cu}\right.$ (1-Pyrbpy)(xantphos)][ $\left.\mathrm{PF}_{6}\right]$, a second set of signals with much lower intensity with respect to the dominant peaks $(\sim 0.05$ : 1.0) was observed. This is shown for $[\mathrm{Cu}(1-$ Pyrbpy)(xantphos)][PF 6$]$ in Fig. 13. For the major and minor components in the spectrum, the signal for $\mathrm{H}^{\mathrm{A} 6}$ (which exhibits a characteristic coupling constant of $\sim 5 \mathrm{~Hz}$ ) appears at $\delta 6.65$ and $8.69 \mathrm{ppm}$, respectively. These values are similar to those observed in $[\mathrm{Cu}(\mathrm{Phbpy})(\mathrm{xantphos})]\left[\mathrm{PF}_{6}\right] \quad(\delta \quad 6.35$ and 8.42 $\mathrm{ppm})^{8}$ (see above) and are consistent with the presence of two conformers of $[\mathrm{Cu}(1-\mathrm{Pyrbpy})(\mathrm{xantphos})]^{+}$which interconvert through inversion of the xanthene 'bowl'. EXSY cross-peaks observed in the ROESY spectrum (Fig. S2 $\dagger$ ) confirmed an exchange process. As we discussed in detail $^{8}$ for $[\mathrm{Cu} \text { (Phbpy)(xantphos) }]^{+}$, the methyl region of the ${ }^{1} \mathrm{H}$ NMR spectrum is very informative. The boat conformation (Fig. 4 and 6$)^{8,34}$ of the xanthene unit forces one methyl group to be in the plane of the xantphos ligand backbone, whereas the second methyl is out-of-plane. This is clear in the projection of the $[\mathrm{Cu}(1-\mathrm{Pyrbpy})(\mathrm{xantphos})]^{+}$cation in Fig. 7. Interconversion of the conformers (Fig. 1c) places the two methyl groups in different magnetic environments. In the dominant conformer, signals for the two Me groups are at $\delta 2.03$ and $1.28 \mathrm{ppm}$, whereas in the second conformer, the corresponding resonances are $\delta 1.14$ and 1.87 ppm, EXSY cross-peaks ( $\delta 1.14$ with 2.03 ppm, and $\delta 1.87$ with $1.28 \mathrm{ppm})$ confirmed exchange. These observations are consistent with those described $\left[\mathrm{Cu}(\mathrm{Phbpy})(\right.$ xantphos) $]\left[\mathrm{PF}_{6}\right]{ }^{8} \quad$ The significantly different populations of the conformers in the case of $[\mathrm{Cu}$ (Phbpy)(xantphos) $]\left[\mathrm{PF}_{6}\right] \quad(\sim 1.0 \quad: \quad 0.9)$ versus $[\mathrm{Cu}(1-$ 
Pyrbpy)(xantphos)][ $\left.\mathrm{PF}_{6}\right](\sim 0.05: 1.0)$ is consistent with the sterically more demanding pyrenyl group.

The room temperature ${ }^{31} \mathrm{P}$ spectra of $[\mathrm{Cu}(2-$ Naphbpy)(POP)][PF $]$ and $[\mathrm{Cu}(2-\mathrm{Naphbpy})(\mathrm{xantphos})]\left[\mathrm{PF}_{6}\right]$ show a broad signals at $\delta-13.4$ and $-12.8 \mathrm{ppm}$, respectively. A similar broad signal at $\delta-14.5 \mathrm{ppm}$ is observed for $[\mathrm{Cu}(1-$ Pyrbpy)(POP) $]\left[\mathrm{PF}_{6}\right]$ at $238 \mathrm{~K}$. In contrast, $[\mathrm{Cu}(1-$ Pyrbpy)(xantphos)][PF 6 shows two doublets at $238 \mathrm{~K}\left({ }^{2} J_{\mathrm{PP}}=\right.$ $95 \mathrm{~Hz})$ at $\delta-10.9$ and $-13.5 \mathrm{ppm}$. A similar pattern is observed in the low temperature spectrum of $[\mathrm{Cu}(1-$ Naphbpy)(xantphos)][PF 6$]\left({ }^{2} J_{\mathrm{PP}}=110 \mathrm{~Hz}\right)$. Two ${ }^{31} \mathrm{P}$ NMR signals $(\delta-12.7$ and $-15.5 \mathrm{ppm})$ are observed for $[\mathrm{Cu}(1-$ Naphbpy)(POP) $]\left[\mathrm{PF}_{6}\right]$ at $253 \mathrm{~K}$, but the coupling could not be resolved. These observations are consistent with desymmetrization of the $\mathrm{P}^{\wedge} \mathrm{P}$ ligand on the ${ }^{1} \mathrm{H}$ NMR time-scale at low temperatures.

\section{Electrochemistry}

The $\left[\mathrm{Cu}\left(\mathrm{N}^{\wedge} \mathrm{N}\right)\left(\mathrm{P}^{\wedge} \mathrm{P}\right)\right]\left[\mathrm{PF}_{6}\right]$ complexes are redox active and cyclic voltammetry was used to investigate the processes. Each complex undergoes a reversible copper-centred oxidation (Table 3 ), and the $E_{1 / 2}{ }^{\text {ox }}$ values are similar to those of +0.82 and $+0.81 \mathrm{~V}$ reported for $\left[\mathrm{Cu}\left(6,6^{\prime}-\mathrm{Me}_{2} \mathrm{bpy}\right)(\mathrm{POP})\right]\left[\mathrm{BF}_{4}\right]$ and $\left[\mathrm{Cu}\left(6,6-\mathrm{Me}_{2}\right.\right.$ bpy)(xantphos) $]\left[\mathrm{BF}_{4}\right] \quad(\mathrm{MeCN}$ solution, vs. $\left.\mathrm{Fc} / \mathrm{Fc}^{+}\right) .{ }^{35}$ For each compound, reduction processes within the solvent accessible window were very poorly defined.

Table 3. Cyclic voltammetric data for $\left[\mathrm{Cu}\left(\mathrm{N}^{\wedge} \mathrm{N}\right)\left(\mathrm{P}^{\wedge} \mathrm{P}\right)\right]\left[\mathrm{PF}_{6}\right]$ complexes referenced to internal $\mathrm{Fc} / \mathrm{Fc}^{+}=0 \mathrm{~V} ; \mathrm{CH}_{2} \mathrm{Cl}_{2}$ (freshly distilled) solutions with $\left[{ }^{\mathrm{n}} \mathrm{Bu}_{4} \mathrm{~N}\right]\left[\mathrm{PF}_{6}\right]$ as supporting electrolyte and scan rate of $0.1 \mathrm{~V} \mathrm{~s}^{-1}$.

$\begin{array}{cc}\text { Complex cation } & E_{1 / 2}{ }^{\text {ox }} / \mathrm{V}\left(E_{\mathrm{pc}}-E_{\mathrm{p}} / \mathrm{mV}\right) \\ {[\mathrm{Cu}(1-\mathrm{Naphbpy})(\mathrm{POP})]^{+}} & +0.79(97) \\ {[\mathrm{Cu}(1-\mathrm{Naphbpy})(\mathrm{xantphos})]^{+}} & +0.82(101) \\ {[\mathrm{Cu}(2-\mathrm{Naphbpy})(\mathrm{POP})]^{+}} & +0.77(96) \\ {[\mathrm{Cu}(2-\mathrm{Naphbpy})(\text { xantphos })]^{+}} & +0.81(101) \\ {[\mathrm{Cu}(1-\mathrm{Pyrbpy})(\mathrm{POP})]^{+}} & +0.77(99) \\ {[\mathrm{Cu}(1-\mathrm{Pyrbpy})(\text { xantphos })]^{+}} & +0.82(88) \\ & \end{array}$

\section{Solution absorption and emission properties}

The solution absorption spectra of $\left[\mathrm{Cu}\left(\mathrm{N}^{\wedge} \mathrm{N}\right)(\mathrm{xantphos})\right]\left[\mathrm{PF}_{6}\right]$ and $\left[\mathrm{Cu}\left(\mathrm{N}^{\wedge} \mathrm{N}\right)(\mathrm{POP})\right]\left[\mathrm{PF}_{6}\right]$ are shown in Fig. 14. The intense absorption bands below $340 \mathrm{~nm}$ correspond to spin-allowed ligand-centred $\quad \pi^{*} \leftarrow \pi \quad$ transitions. For $[\mathrm{Cu}(1-$ Naphbpy $\left.)\left(\mathrm{P}^{\wedge} \mathrm{P}\right)\right]\left[\mathrm{PF}_{6}\right]$ and $\left[\mathrm{Cu}(2-\mathrm{Naphbpy})\left(\mathrm{P}^{\wedge} \mathrm{P}\right)\right]\left[\mathrm{PF}_{6}\right]\left(\mathrm{P}^{\wedge} \mathrm{P}=\right.$ POP or xantphos), a broad absorption of low intensity appears at $\sim 390 \mathrm{~nm}$ arising from metal-to-ligand charge transfer (MLCT). For the complexes containing 1-Pyrbpy (dotted curves in Fig. 14), the profile of the bands between 225 and $\sim 335 \mathrm{~nm}$ reflects that of the absorption spectrum of the free 1Pyrbpy ligand (Fig. S3 $\dagger$ ) which is turn is similar to, but broader than, that of pyrene. We assign the broad absorption around 350 $\mathrm{nm}$ to a charge-transfer from the pyrenyl to bpy unit. ${ }^{36,37}$

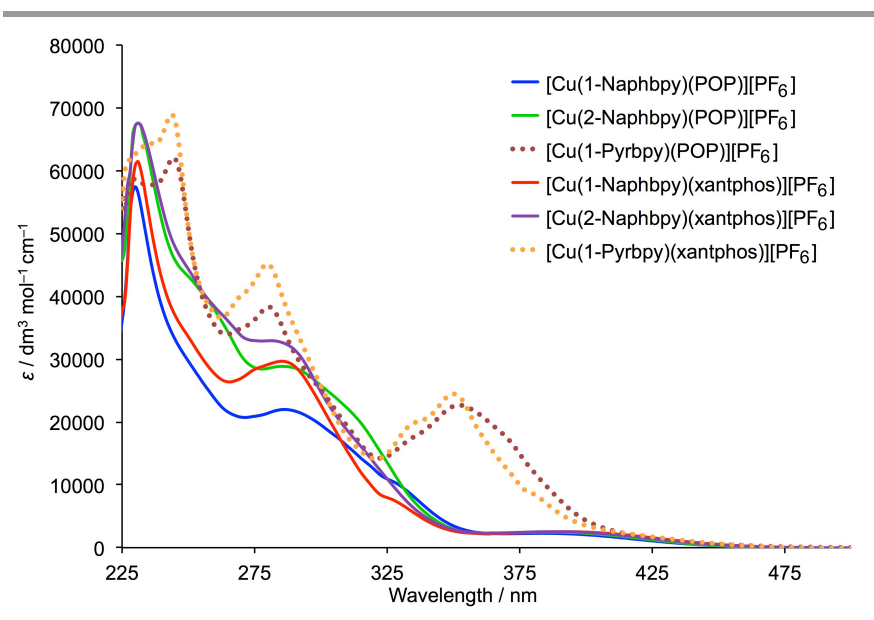

Fig. 14. Absorption spectra of the $\left[\mathrm{Cu}\left(\mathrm{N}^{\wedge} \mathrm{N}\right)\left(\mathrm{P}^{\wedge} \mathrm{P}\right)\right]\left[\mathrm{PF}_{6}\right]$ complexes $\left(\mathrm{CH}_{2} \mathrm{Cl}_{2}, 2.5 \times\right.$ $10^{-5} \mathrm{~mol} \mathrm{dm}^{-3}$ ).

Dichloromethane solutions of the $\left[\mathrm{Cu}\left(\mathrm{N}^{\wedge} \mathrm{N}\right)\left(\mathrm{P}^{\wedge} \mathrm{P}\right)\right]\left[\mathrm{PF}_{6}\right]$ complexes were poorly emissive. Emission data for powder samples are given in Table 4. The complexes are all orange emitters with values of $\lambda_{\max }{ }^{\text {em }}$ in the range 582 to $617 \mathrm{~nm}$. The emission spectra of the complexes containing 1-Naphby and 2Naphbpy are shown in Fig. 15. Photoluminescence quantum yileds (PLQY) were $<6 \%$ and the emission lifetimes $\left(\tau_{1 / 2}\right)$ were all $\sim 2 \mu$ s (Table 4). Both $[\mathrm{Cu}(1-\mathrm{Pyrbpy})(\mathrm{POP})]\left[\mathrm{PF}_{6}\right]$ and $[\mathrm{Cu}(1-\mathrm{Pyrbpy})(\mathrm{xantphos})]\left[\mathrm{PF}_{6}\right]$ are extremely weak emitters in the solid state. This may be due to a low lying triplet state which leads to quenching of the MLCT emission.

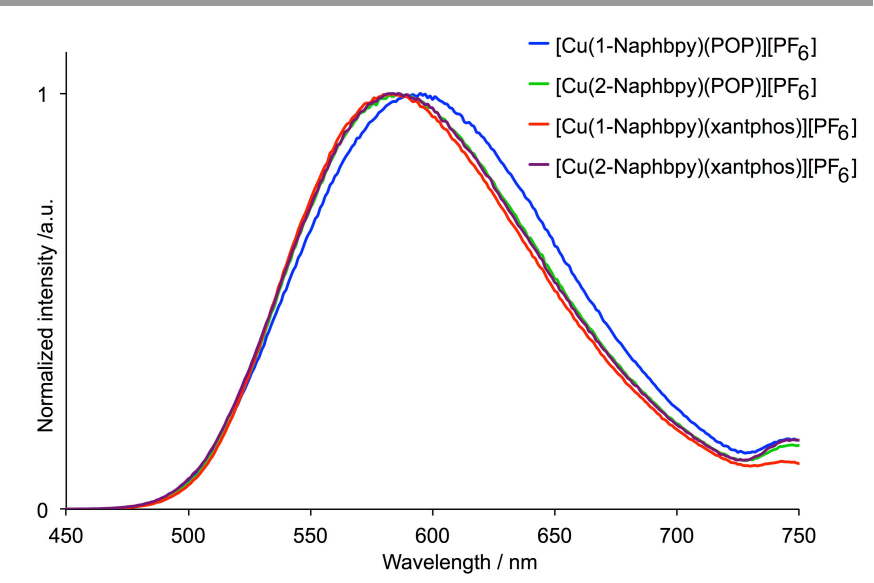

Fig. 15. Emission spectra of powder samples of $\left[C u\left(N^{\wedge} N\right)\left(P^{\wedge} P\right)\right]\left[P F_{6}\right]$ complexes containin 1-Naphby and 2-Naphbpy $\left(\lambda_{\text {exc }}=365 \mathrm{~nm}\right)$. 
Table 4. Emission maxima and PLQY for powder samples of $\left[\mathrm{Cu}\left(\mathrm{P}^{\wedge} \mathrm{P}\right)\left(\mathrm{N}^{\wedge} \mathrm{N}\right)\right]\left[\mathrm{PF}_{6}\right]$ complexes $\left(\lambda_{\mathrm{exc}}=365 \mathrm{~nm}\right)$.

\begin{tabular}{|c|c|c|c|c|c|}
\hline Compound & $\begin{array}{c}\lambda_{\mathrm{em}}{ }^{\max } \\
/ \mathrm{nm}\end{array}$ & $\begin{array}{l}\text { PLQY } \\
/ \%\end{array}$ & $\begin{array}{l}\tau_{1 / 2}(1) / \mu \mathrm{s} \\
(\mathrm{A} 1)\end{array}$ & $\begin{array}{l}\tau_{1 / 2}(2) / \mu \mathrm{s} \\
(\mathrm{A} 2)\end{array}$ & $\tau_{1 / 2}(\mathrm{av}) / \mu \mathrm{s}$ \\
\hline $\begin{array}{c}\mathrm{Cu}(1- \\
\text { Naphbpy)(POP) }]\left[\mathrm{PF}_{6}\right]\end{array}$ & 595 & 4.0 & $\begin{array}{c}2.9 \\
(0.3812) \\
\end{array}$ & $\begin{array}{c}1.1 \\
(0.4664)\end{array}$ & $1.9^{a}$ \\
\hline $\begin{array}{c}{[\mathrm{Cu}(2-} \\
\text { Naphbpy)(POP)][PF } 6] \\
\end{array}$ & 586 & 4.6 & $\begin{array}{c}2.5 \\
(0.4636) \\
\end{array}$ & $\begin{array}{c}0.8 \\
(0.4101)\end{array}$ & $1.7^{a}$ \\
\hline$[\mathrm{Cu}(1-\mathrm{Pyrbpy})(\mathrm{POP})]\left[\mathrm{PF}_{6}\right]$ & 617 & 0.3 & & & ${ }^{b}$ \\
\hline $\begin{array}{c}{[\mathrm{Cu}(1-} \\
\text { Naphbpy)(xantphos) })\left[\mathrm{PF}_{6}\right]\end{array}$ & 582 & 5.6 & $\begin{array}{c}2.9 \\
(0.4547)\end{array}$ & $\begin{array}{c}1.1 \\
(0.3933)\end{array}$ & $2.1^{a}$ \\
\hline $\begin{array}{c}{[\mathrm{Cu}(2-} \\
\left.\text { Naphbpy)(xantphos)][} \mathrm{PF}_{6}\right]\end{array}$ & 586 & 3.6 & $\begin{array}{c}2.7 \\
(0.4644) \\
\end{array}$ & $\begin{array}{c}1.0 \\
(0.3951) \\
\end{array}$ & $1.9^{a}$ \\
\hline $\begin{array}{c}{[\mathrm{Cu}(1-} \\
\text { Pyrbpy)(xantphos)][PF }]\end{array}$ & 576 & 0.3 & & & \\
\hline
\end{tabular}

${ }^{a}$ Biexponential fit using the equation $\tau_{1 / 2}(\mathrm{av})=\sum \mathrm{A}_{\mathrm{i}} \tau_{\mathrm{i}} / \sum \mathrm{A}_{\mathrm{i}}$ where $\mathrm{A}_{\mathrm{i}}$ is the pre-exponential factor for the lifetime. ${ }^{b}$ Not measured.

\section{Conclusions}

We have prepared a series of $\left[\mathrm{Cu}\left(\mathrm{N}^{\wedge} \mathrm{N}\right)(\mathrm{POP})\right]\left[\mathrm{PF}_{6}\right]$ and $\left[\mathrm{Cu}\left(\mathrm{N}^{\wedge} \mathrm{N}\right)(\right.$ xantphos) $]\left[\mathrm{PF}_{6}\right]$ compounds in which $\mathrm{N}^{\wedge} \mathrm{N}$ is a bpy ligand bearing a sterically hindered 1-naphthyl, 2-naphthyl or 1-pyrenyl substituent in the 6-position. Crystallographic data confirm that the copper(I) centre is in a distorted tetrahedral environment, and the $\mathrm{N}^{\wedge} \mathrm{N}$ ligand is oriented with the stericallydemanding aryl substituent positioned away from the $\left(\mathrm{C}_{6} \mathrm{H}_{4}\right)_{2} \mathrm{O}$ unit of POP or the xanthene 'bowl' of xantphos. Each POPcontaining complex exhibits face-to-face $\pi$-stacking between one arene ring of the $\left(\mathrm{C}_{6} \mathrm{H}_{4}\right)_{2} \mathrm{O}$ unit and one phenyl ring of a $\mathrm{PPh}_{2}$ unit. Despite the extended $\pi$-systems, intermolecular faceto-face $\pi$-contacts do not play a dominant role in the packing of the compounds in the solid state.

In solution, the complexes undergo dynamic behaviour, and the ${ }^{1} \mathrm{H}$ NMR signals from the POP and xantphos ligands are broad to very broad at $298 \mathrm{~K}$. At low temperatures, the phenyl rings in each $\mathrm{PPh}_{2}$ group in the POP-containing compounds gives two sets of signals (pointing towards or away from the 6substituted aryl group on the bpy) but exchange associated with the conformationally flexible 8-membered chelate $\{\mathrm{CuPCCOCCP}\}$-ring occurs at higher temperatures; this was confirmed using EXSY exchange spectroscopy. In acetone solution, the $\left[\mathrm{Cu}\left(\mathrm{N}^{\wedge} \mathrm{N}\right)(\mathrm{xantphos})\right]^{+}$complexes exist as a mixture of conformers which interconvert through inversion of the xanthene 'bowl' (Fig. 1). Proton $\mathrm{H}^{\mathrm{A} 6}$ resides in very different magnetic environments in the two conformers, and the ratio of conformers in $[\mathrm{Cu}(1-\mathrm{Pyrbpy})(\mathrm{xantphos})]\left[\mathrm{PF}_{6}\right]$ is $\sim 0.05: 1.0$. This compares to almost equal populations for the analogous conformers of $[\mathrm{Cu}(\mathrm{Phbpy})(\text { xantphos })]^{+}$, demonstrating the significant steric influences of going from a phenyl to 1-pyrenyl 6-substituent.

Consistent with the trends previously observed on going from 6-alkyl to 6-phenyl substituents in the bpy ligand in $\left[\mathrm{Cu}\left(\mathrm{N}^{\wedge} \mathrm{N}\right)(\mathrm{POP})\right]^{+}$and $\left[\mathrm{Cu}\left(\mathrm{N}^{\wedge} \mathrm{N}\right)(\mathrm{xantphos})\right]^{+}$complexes, ${ }^{8}$ the introduction of the 1-Naphthyl or 2-Naphthyl substituents result in poor PLQY values. In the case of the 1-Pyrbpy ligand, a low lying triplet state may be responsible for quenching the MLCT emission.

\section{Acknowledgements}

We acknowledge the Swiss National Science Foundation (Grant number 162631) and the University of Basel for financial support. We thank Sarah Keller for invaluable suggestions.

\section{Notes and references}

${ }^{a}$ Department of Chemistry, University of Basel, Spitalstrasse 51, CH4056 Basel, Switzerland; email: catherine.housecroft@unibas.ch $\dagger$ Electronic Supplementary Information (ESI) available: CCDC 15285481528552. Fig. S1-S2: additional NMR sepectra; Fig. S3: absorption spectrum of 1-Pyrbpy. See DOI: 10.1039/b000000x/

1 F. Dumur, Org. Electronics, 2015, 21, 27

2 R. D. Costa, E. Ortí, H. J. Bolink, F. Monti, G. Accorsi and N. Armaroli, Angew. Chem. Int. Ed., 2012, 51, 8178.

3 M. Magni, P. Biagini, A. Colombo, C. Dragonetti, D. Roberto and A. Valore, Coord. Chem. Rev., 2016, 322, 69.

4 D. G. Cuttell, S.-M. Kuang, P. E. Fanwick, D. R. McMillin and R. A. Walton, J. Am. Chem. Soc., 2002, 124, 6.

5 S.-M. Kuang, D. G. Cuttell, D. R. McMillin, P. E. Fanwick and R. A. Walton, Inorg. Chem., 2002, 41, 3313.

6 R.D. Costa, D. Tordera, E. Ortí, H.J. Bolink, J. Schönle, S. Graber, C.E. Housecroft, E.C. Constable and J.A. Zampese, J. Mater. Chem. 2011, 21, 16108.

7 S. Keller, E. C. Constable, C. E. Housecroft, M. Neuburger, A. Prescimone, G. Longo, A. Pertegás, M. Sessolo and H. J. Bolink, Dalton Trans., 2014, 43, 16593.

8 S. Keller, A. Pertegás, G. Longo, L. Martinez, J. Cerdá, J.M. Junquera-Hernández, A. Prescimone, E. C. Constable, C. E. Housecroft, E. Ortí and H. J. Bolink, J. Mater. Chem. C, 2016, 4, 3857.

9 C. Bizzarri, C. Strabler, J. Prock, B. Trettenbrein, M. Ruggenthaler, C.-H. Yang, F. Polo, A. Iordache, P. Brüggeller and L. De Cola, Inorg. Chem., 2014, 53, 10944.

10 M.D. Weber, C. Garino, G. Volpi, E. Casamassa, M. Milanesio, C. Barolo and R.D. Costa, Dalton Trans., 2016, 45, 8984.

11 F. Brunner, L. Martínez-Sarti, S. Keller, A. Pertegás, A. Prescimone, E.C. Constable, H.J. Bolink and C.E. Housecroft, Dalton Trans., 2016, 45, 15180.

12 R. Czerwieniec and H. Yersin, Inorg. Chem., 2015, 54, 4322.

13 D. Asil, J. A. Foster, A. Patra, X. de Hatten, J. del Barrio, O. A. Scherman, J.R. Nitschke and R. H. Friend, Angew. Chem. Int. Ed., 2014, 53, 8388 . 
14 N. Armaroli, G. Accorsi, M. Holler, O. Moudam, J.-F. Nierengarten, Z. Zhou, R.T. Wegh and R. Welter, Adv. Mater., 2006, 18, 1313.

15 L. Zhang, B. Li and Z. Su, J. Phys. Chem. C, 2009, 113, 13968.

16 C L. Linfoot, M. J. Leitl, P. Richardson, A. F. Rausch, O. Chepelin, F. J. White, H. Yersin and N. Robertson, Inorg. Chem., 2014, 53, 10854 .

17 S. Medina-Rodríguez, F.J. Orriach-Fernández, C. Poole, P. Kumar, A. de al Torre-Vega, J.F. Fernández-Sánchez, E. Baranoff and A. Fernández-Gutiérrez, Chem. Commun., 2015, 51, 11401.

18 H. Takeda, K. Ohashi, A. Sekine and O. Ishitani, J. Am. Chem. Soc., 2016, 138, 4354.

19 A.J.J. Lennox, S. Fischer, M. Jurrat, S.-P. Luo, N. Rockstroh, H. Junge, R. Ludwig and M. Beller, Chem. Eur. J., 2016, 22, 1233.

20 S.-P. Luo, N.-Y. Chen, Y.-Y. Sun, L.-M. Xia, Z.-C. Wu, H. Junge, M. Beller and Q.-A. Wu, Dyes Pigments, 2016, 134, 580.

21 A. Kaeser, M. Mohankumar, J. Mohanraj, F. Monti, M. Holler, J.-J. Cid, O. Moudam, I. Nierengarten, L. Karmazin-Brelot, C. Duhayon, B. Delavaux-Nicot, N. Armaroli and J.-F. Nierengarten, Inorg. Chem., 2013, 52, 12140

22 T. Norrby, A. Börje, L. Zhang and B. Akermark, Acta Chem. Scand., 1998, 52, 77

23 G. J. Kubas, Inorg. Synth., 1979, 19, 90

24 Y. J. Pu, M. Miyamoto, K. Nakayama, T. Oyama, Y. Masaaki and J. Kido, Org. Electronics, 2009, 10, 228.

25 G. E. Schneider, A. Pertegás, E. C. Constable, C. E. Housecroft, N. Hostettler, C. D. Morris, J. A. Zampese, H. J. Bolink, J. M. JunqueraHernández, E. Ortí and M. Sessolo, J. Mater. Chem. C, 2014, 2, 7047.

26 Bruker Analytical X-ray Systems, Inc., 2006, APEX2, version 2 User Manual, M86-E01078, Madison, WI.

27 P. W. Betteridge, J. R. Carruthers, R. I. Cooper, K. Prout and D. J. Watkin, J. Appl. Cryst., 2003, 36, 1487.

28 I. J. Bruno, J. C. Cole, P. R. Edgington, M. K. Kessler, C. F. Macrae, P. McCabe, J. Pearson, R. Taylor, Acta Crystallogr., Sect. B, 2002, 58, 389.

29 C. F. Macrae, I. J. Bruno, J. A. Chisholm, P. R. Edgington, P. McCabe, E. Pidcock, L. Rodriguez-Monge, R. Taylor, J. van de Streek and P. A. Wood, J. Appl. Cryst., 2008, 41, 466.

30 J.G. Park and Y. Jahng, Bull. Korean Chem. Soc., 1998, 19, 436.

31 J. Yuasa, M. Dan and T. Kawai, Dalton Trans., 2013, 42, 16096

32 M. Nishio, CrystEngComm, 2004, 6, 130.

33 See for example: G. Tárkányi, P. Király, G. Pálinkás and A. Deák, Mag. Res. Chem., 2007, 45, 917; A. Pintado-Alba, H. de la Riva, M. Nieuwhuyzen, D. Bautista, P. R. Raithby, H. A. Sparkes, S. J. Teat, J.
M. López-de-Luzuriaga and M. C. Lagunas, Dalton Trans., 2004, 3459.

34 S. Keller, F. Brunner, A. Prescimone, E. C. Constable and C. E. Housecroft, Inorg. Chem. Comm., 2015, 58, 64 and references cited therein.

35 I. Andrés-Tomé, J. Fyson, F. Baiao Dias, A. P. Monkman, G. Iacobellis and P. Coppo, Dalton Trans., 2012, 41, 8669.

36 E. C. Constable, C. E. Housecroft, M. Neuburger, P. Rösel, G.E. Schneider, J. A. Zampese, F. Monti, N. Armaroli, R. D. Costa and E. Ortí, Inorg. Chem., 2013, 52, 885.

37 A. Harriman and M. Hissler, Phys. Chem. Chem. Phys., 1999, 1, 4203 . 